\title{
Colors of an Earth-like exoplanet
}

\section{Temporal flux and polarization signals of the Earth}

\author{
A. $\operatorname{Groot}^{1}$, L. Rossi ${ }^{2}$, V. J. H. Trees ${ }^{1, \star}$, J. C. Y. Cheung ${ }^{1}$, and D. M. Stam ${ }^{1}$ \\ ${ }^{1}$ Faculty of Aerospace Engineering, Delft University of Technology, Delft, The Netherlands \\ e-mail: d.m.stam@tudelft.nl \\ ${ }^{2}$ LATMOS/IPSL, UVSQ Université Paris-Saclay, Sorbonne Université, CNRS, Guyancourt, France
}

Received 24 January 2020 / Accepted 12 June 2020

\begin{abstract}
Context. Understanding the total flux and polarization signals of Earth-like planets and their spectral and temporal variability is essential for the future characterization of such exoplanets.

Aims. We provide computed total $(F)$ and linearly $(Q$ and $U)$ and circularly $(V)$ polarized fluxes, and the degree of polarization $P$ of sunlight that is reflected by a model Earth, to be used for instrument designs, optimizing observational strategies, and/or developing retrieval algorithms.

Methods. We modeled a realistic Earth-like planet using one year of daily Earth-observation data: cloud parameters (distribution, optical thickness, top pressure, and particle effective radius), and surface parameters (distribution, surface type, and albedo). The Stokes vector of the disk-averaged reflected sunlight was computed for phase angles $\alpha$ from $0^{\circ}$ to $180^{\circ}$, and for wavelengths $\lambda$ from 350 to $865 \mathrm{~nm}$.

Results. The total flux $F$ is one order of magnitude higher than the polarized flux $Q$, and $Q$ is two and four orders of magnitude higher than $U$ and $V$, respectively. Without clouds, the peak-to-peak daily variations due to the planetary rotation increase with increasing $\lambda$ for $F, Q$, and $P$, while they decrease for $U$ and $V$. Clouds modify but do not completely suppress the variations that are due to rotating surface features. With clouds, the variation in $F$ increases with increasing $\lambda$, while in $Q$, it decreases with increasing $\lambda$, except at the largest phase angles. In earlier work, it was shown that with oceans, $Q$ changes color from blue through white to red. The $\alpha$ where the color changes increases with increasing cloud coverage. Here, we show that this unique color change in $Q$ also occurs when the oceans are partly replaced by continents, with or without clouds. The degree of polarization $P$ shows a similar color change. Our computed fluxes and degree of polarization will be made publicly available.
\end{abstract}

Key words. radiative transfer - polarization - techniques: polarimetric - planets and satellites: terrestrial planets planets and satellites: atmospheres - planets and satellites: surfaces

\section{Introduction}

Since the discovery of 51 Pegasi b (Mayor \& Queloz 1995), several thousand exoplanets have been discovered and confirmed ${ }^{1}$, including various Earth-sized planets in the habitable zones around their parent stars (Chou et al. 2017). A recent addition to the latter selection is TOI-700b, an Earth-sized planet in the habitable zone of red dwarf star TOI-700, as discovered by Gilbert et al. (2020) and confirmed with Spitzer observations by Rodriguez et al. (2020).

The planetary physical properties that can be derived from measurements with the two most successful exoplanet detection techniques, that is, with the radial velocity method and the transit method, are the planet radius (if the planet transits its star), mass (a lower limit if the planet does not transit its star), and the orbital period and distance, eccentricity, and inclination (if the planet transits its star) (see Perryman 2018, and references therein). The upper atmospheres (above optically thick clouds) of giant exoplanets and those of smaller planets around $\mathrm{M}$ stars can be probed using spectroscopy during primary and/or secondary transits (Bean et al. 2010; Swain et al. 2009, 2008; Tinetti

\footnotetext{
^ Now at Royal Netherlands Meteorological Institute (KNMI), De Bilt, The Netherlands.

1 http://exoplanetarchive.ipac. caltech. edu
}

et al. 2007; Nakajima 1983). It appears to be virtually impossible to characterize the (lower) atmospheres and surfaces of small, Earth-like planets in the habitable zones of solar-type stars (Bétrémieux \& Kaltenegger 2014; Misra et al. 2014; Kaltenegger $\&$ Traub 2009), in particular because the light of the parent star is refracted while traveling through the lower atmosphere of its planet and emerges forever out of reach of terrestrial telescopes (García Muñoz et al. 2012). The (lower) atmosphere and surface of a planet are crucial for determining the habitability of a planet, as they hold information about cloud composition, trace gases in disequilibrium and probably most importantly, liquid surface water (see, e.g., Schwieterman et al. 2018; Kiang et al. 2007a,b, and references therein). For such a characterization of terrestrial-type planets, direct observations of the thermal radiation that they emit or of the light of their parent star that they reflect are required. The numerical results that we present in this paper concern the reflected starlight. Because of the huge distances involved, any measured reflected starlight pertains to the (illuminated and visible part of the) planetary disk. It therefore is a disk-integrated signal.

Ford et al. (2001) showed with numerical simulations that the daily rotation of the Earth induces repetitive patterns in the disk-integrated flux that is reflected by the Earth observed from afar because of the continents and oceans and the clouds. 
Stam (2008) showed how not only the total flux of reflected sunlight, but also the polarized fluxes and the degree of polarization of this light vary as an Earth-like planet rotates and orbits its star. Recently, Trees \& Stam (2019) showed the change in the color of the polarized fluxes and degree of polarization throughout the orbit when an ocean is present. Polarimetry indeed appears to be a powerful tool for the detection and characterization of exoplanets. In particular, when it is integrated over the stellar disk, direct starlight is virtually unpolarized (Kemp et al. 1987), while starlight that has been scattered in a planetary atmosphere and/or has been reflected by a planetary surface is usually polarized. Polarimetry thus enhances the contrast between a star and an orbiting exoplanet (by three to five orders of magnitude, according to Keller et al. 2010), allows the direct confirmation of the nature of the substellar companion, and the state of polarization of the reflected light holds information about the composition and structure of the planetary atmosphere and surface (if present) (see Wiktorowicz \& Stam 2015, for details and references).

A recent polarimetric detection in reflected light of a substellar companion that is possibly surrounded by a disk was announced by Ginski et al. (2018). The recent tentative polarimetric detection of banded, that is, Jupiter-like, cloud structures on Luhman 16A by Millar-Blanchaer et al. (2020) supports the application of polarimetry for exoplanet characterization (in this case in the thermally emitted signal of this binary brown dwarf system component).

In this paper, we present the computed phase curves of the total and polarized fluxes and the degree of polarization of starlight that is reflected by an Earth-like exoplanet with a spatially and temporally changing cloud and surface cover, all based on daily data from Earth-observation satellite instruments. The temporal resolution of our computations is $15 \mathrm{~min}$, and it covers 2011 completely. The phase angle ranges from $0^{\circ}$ to $180^{\circ}$. We did not include gaseous absorption, and instead concentrated on the broadband continuum fluxes at 350, 443, 550, 670, and $865 \mathrm{~nm}$.

Our computed phase curves are publicly available and can be used for the design of future instruments for the detection and characterization of terrestrial-type exoplanets. The polarization state of reflected starlight also needs to be included for the design of instruments that aim at measuring only total fluxes, because the response of optical elements such as mirrors and gratings usually depends on the polarization state of the incident light. The curves can also be used for the development and testing of retrieval algorithms (see, e.g., Aizawa et al. 2020; Fan et al. 2019; Berdyugina \& Kuhn 2019; Kawahara \& Fujii 2011), and for the optimization of observational strategies. A number of references describing the use of terrestrial remote-sensing satellite measurements of the total flux of sunlight reflected by Earth for the testing of retrieval algorithms are provided by Jiang et al. (2018). Fluxes that have been measured in this way, however, usually do not cover the phase angle range and/or do not have the temporal resolution and/or do not cover the spectral range to fully assess (future) measurements of exoplanet fluxes. Earth-shine measurements (see Turnbull et al. 2006; Woolf et al. 2002, and references to these papers), where the sunlight that is reflected by the Earth is detected on the nightside of the moon, are also limited by the phase angle range and the lack of knowledge of the reflection properties of the lunar surface. Computed reflected fluxes do not suffer from these limitations, and the user has the ability to add specific noise and/or instrumental effects.

As far as we know, the polarized fluxes of the (distant) Earth have never been measured directly. Linearly polarized fluxes of local regions on Earth have been measured by the POLDER-3/
PARASOL Low-Earth-Orbit satellite instrument (Fougnie et al. 2007) and its predecessors POLDER-1 and POLDER-2 (Deschamps et al. 1994). Dubovik et al. (2019) presented an excellent review of the characterization of aerosol and surface properties from such local data. Attempts have been made to translate local POLDER-3/PARASOL data into a polarized signal that simulates the disk-integrated signal of the Earth (Wang et al. 2019), but especially because polarization signals depend strongly on the illumination and viewing geometries (see, e.g., Hansen \& Travis 1974), the results of these attempts remain very limited. First spectropolarimetric measurements of Earth-shine have been presented by Miles-Páez et al. (2014), Bazzon et al. (2013), and Sterzik et al. (2012), and a more in-depth analysis of the latter data can be found in Sterzik et al. (2019) and Emde et al. (2017). In these measurements, the reflection properties of the lunar surface, in particular, the reflection of polarized incident Earth-shine by the lunar surface, indeed remains a source of uncertainty.

A few instrument developments have aimed at measuring total and polarized fluxes of sunlight that is reflected by the Earth from afar, such as the Lunar Observatory of Unresolved Polarimetry of Earth (LOUPE; Hoeijmakers et al. 2016; Karalidi et al. 2012a) that is scheduled to observe the Earth from the surface of the moon (or, e.g., a geostationary satellite, which would mean that we would not be able to monitor the daily rotation of the Earth, but which would ensure continuous access to power). Examples of future telescope instruments that are designed to measure polarization of exoplanets are EPOL, the imaging polarimeter of the Exoplanet Imaging Camera and Spectrograph (EPICS) for the European Extremely Large Telescope (E-ELT; Kasper et al. 2010; Keller et al. 2010), and POLLUX, a UV polarimeter, that is envisioned for the NASA Large UV Optical Infrared Surveyor (LUVOIR) space telescope concept (Bouret et al. 2018).

A number of publications has focused on the simulation of reflected-light signals of Earth-like exoplanets (see the various references in this article), but did not have the temporal resolution of our results. Two other important factors in our model are the reflection by the wind-ruffled ocean surface (as described by Trees \& Stam 2019) and the realistic cloud properties. Simulations of light reflected by exo-oceans have been presented by Zugger et al. (2010) and Williams \& Gaidos (2008). They used models without full polarization, with simplified cloud models, and without cloud and surface variability.

The outline of this paper is as follows. In Sect. 2 we describe the numerical algorithm that we used to compute the fluxes and polarization signals of our model planets, where we start with our definitions and end with a concise description of the radiative transfer algorithm. In Sect. 3, we describe the parameters of the model planet and the databases from which we have obtained these parameters. In Sect. 4, we present the computed total and polarized fluxes and degree of polarization for cloud-free and cloudy model planets. Finally, in Sect. 5, we summarize and discuss our results and describe possible improvements of the numerical simulations and the relation with (future) observations.

\section{Numerical algorithms}

\subsection{Definitions of light and polarization}

We describe the starlight that is incident on a planet and the starlight that is reflected by the planet by the Stokes vector $\boldsymbol{F}$ 
(see, e.g., Hovenier et al. 2004; Hansen \& Travis 1974),

$\boldsymbol{F}=\left[\begin{array}{c}F \\ Q \\ U \\ V\end{array}\right]$,

where $F$ is the total flux, $Q$ and $U$ are the linearly polarized fluxes, and $V$ is the circularly polarized flux (all in $\mathrm{W} \mathrm{m}^{-2}$, or $\mathrm{W} \mathrm{m}^{-3}$ when defined per wavelength). Fluxes $Q$ and $U$ are defined with respect to the planetary scattering plane, which is the plane through the centers of the star, planet, and the observer. They represent the following flux differences:

$Q=F_{0^{\circ}}-F_{90^{\circ}}$

$U=F_{45^{\circ}}-F_{135^{\circ}}$,

where $F_{x^{\circ}}$ is the flux measured through a linear polarization filter with its optical axis making an angle of $x^{\circ}$ with the reference plane, measured by rotating in the clockwise direction when looking toward the planet (Hovenier et al. 2004; Hansen \& Travis 1974). Fluxes $Q$ and $U$ can be redefined with respect to another reference plane, such as the optical plane of an instrument, using a rotation matrix (see Hovenier \& van der Mee 1983, for the definition). The circularly polarized flux $V$ is positive when the observer sees the electric vector of starlight that is reflected by a planet rotating in the anticlockwise direction, and $V$ is negative when the observer sees a rotation in the clockwise direction.

Light of a solar-type star can be assumed to be unpolarized when integrated over the stellar disk. This assumption is based on observations of the Sun itself by Kemp et al. (1987) and on observations of solar-type stars (active and inactive FGK stars) by Cotton et al. (2017). Anomalies in the symmetry of the stellar disk, such as flares and/or spots, are expected to produce degrees of linear polarization of about $10^{-6}$ (Kostogryz et al. 2015; Berdyugina et al. 2011). We represent the stellar flux vector that is incident on the planet by the (column) vector $\boldsymbol{F}_{0}=F_{0}[1,0,0,0]=F_{0} \mathbf{1}$, where $\pi F_{0}$ is the total flux as measured in a plane perpendicular to the incident direction.

Starlight that has been reflected by a planet is usually polarized because it has been scattered by gases and aerosols or cloud particles in the planetary atmosphere and/or has been reflected by the surface below the atmosphere. The degree of polarization of this light is defined as

$P_{\mathrm{tot}}=\frac{\sqrt{Q^{2}+U^{2}+V^{2}}}{F}$.

The Stokes parameter $V$ of sunlight that is reflected by an Earthlike planet is expected to be very small (see Rossi \& Stam 2018; Kawata 1978; Hansen \& Travis 1974, and results in this article), and $P_{\text {tot }}$ is therefore usually virtually equal to the degree of linear polarization,

$P=\frac{\sqrt{Q^{2}+U^{2}}}{F}$.

Because our Earth-like model planets are horizontally inhomogeneous because they have continents and clouds and are usually asymmetric with respect to the reference plane, the Stokes parameter $U$ (see Eq. (3)) is usually not zero. It appears to be very small, as we show in Sect. 4 . When $U \approx 0$, the sign of $Q$ indicates the direction of $P$ : if $Q>0(Q<0)$, the direction is parallel (perpendicular) to the planetary scattering plane.

\subsection{Radiative transfer algorithm}

We computed the total and polarized fluxes $F, Q, U$, and $V$, and the degree of polarization of starlight that is reflected by a model planet using the Python-Fortran tool PyMieDAP ${ }^{2}$. A detailed description of PyMieDAP is given by Rossi et al. (2018). We summarize the parts that are relevant for the interpretation of our numerical results. We computed reflected flux vectors for spatially resolved model planets. These results apply, for example, to Solar System planets. By integrating the spatially resolved fluxes across the planetary disk, we obtain the spatially unresolved reflected flux vectors, which apply in particular to (future) observations of exoplanets.

For our spatially resolved computations, the 2D planetary disk facing the observer is divided into equal-sized, square pixels. We used 100 pixels along the light equator of the planet (except for the images shown in Figs. 1-2). The actual planetary surface area that is covered by a pixel increases strongly toward the planetary limb. For the center of each pixel, we computed the illumination and viewing angles on the 3D spherical planet (for detailed definitions, see Rossi et al. 2018; de Haan et al. 1987):

Illumination angle $\theta_{0}$. The angle between the local vertical and the direction toward the star.

Viewing angle $\theta$. The angle between the local vertical and the direction toward the observer.

Azimuthal angle $\phi-\phi_{0}$. The difference angle between the plane containing the local vertical and the direction of the incident light, and the plane containing the local vertical and the direction toward the observer.

Angles $\theta_{0}$ and $\phi_{0}$ depend on the planetary phase angle $\alpha$, that is, the angle between the center of the star and the observer as measured from the center of the planet. For a planetary orbit with an orbital inclination angle $i, 90^{\circ}-i \leq \alpha \leq 90^{\circ}+i$. In this paper, we present results for an edge-on orbit, that is, for $i=90^{\circ}$, and $0^{\circ} \leq \alpha \leq 180^{\circ}$. For other inclination angles, the appropriate phase angle range can be chosen from our results.

Given the illumination and viewing geometries, and the atmosphere-surface properties for each pixel, we computed $\boldsymbol{F}$ (Eq. (1)) of the reflected starlight for a given pixel $i$, using (Hansen \& Travis 1974)

$\boldsymbol{F}_{i}\left(\lambda, \theta, \theta_{0}, \phi-\phi_{0}\right)=\cos \theta_{0} \boldsymbol{R}_{i 1}\left(\lambda, \theta, \theta_{0}, \phi-\phi_{0}\right) \boldsymbol{F}_{0}(\lambda)$,

where $\boldsymbol{R}_{i 1}$ is the first column of the local reflection matrix on the planet (only the first column is relevant, because the incoming starlight is assumed to be unpolarized). We computed $\boldsymbol{R}_{i 1}$ using an adding-doubling radiative transfer algorithm in Fortran (de Haan et al. 1987), that fully includes polarization for all orders of scattering. We computed the reflection by horizontally inhomogeneous planets by choosing different atmospheresurface models for different pixels (see Sect. 3). The reflection of each pixel was computed independently from that of its neighbours. Given the physical surface areas covered by the pixels, the contribution of light that scatters from one pixel to another and then toward the observer is negligible.

Rather than embarking on a separate radiative transfer computation for every pixel, we first computed and stored the coefficients $\boldsymbol{R}_{i 1}^{m}\left(\theta, \theta_{0}\right)(0 \leq m<M$, where $M$ is the total number of coefficients) of the expansion of $\boldsymbol{R}_{i 1}\left(\theta, \theta_{0}, \phi-\phi_{0}\right)$ into a

2 PyMieDAP can be downloaded under the GNU GPL license at: https://gitlab.com/loic.cg.rossi/pymiedap 


\section{Total flux $F$}

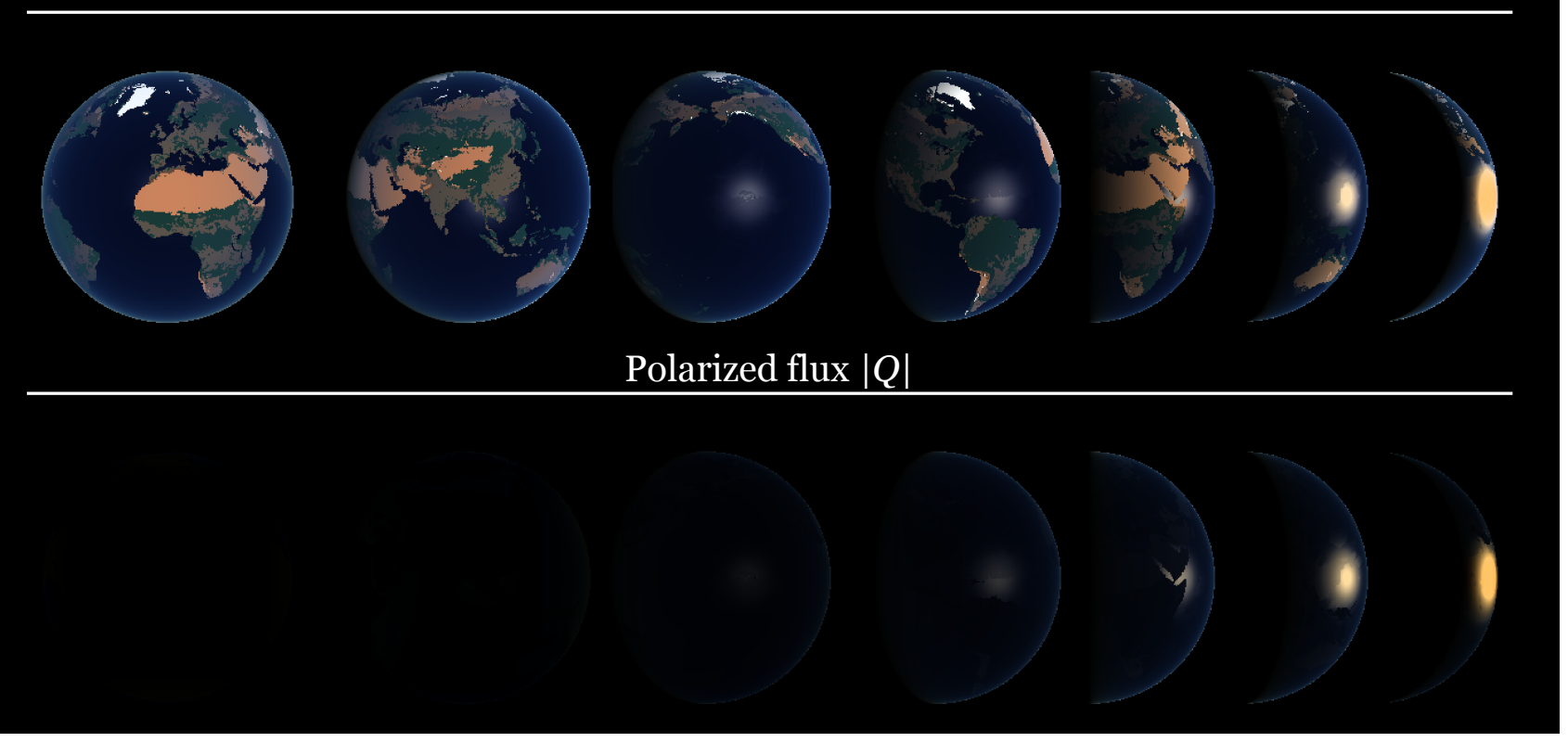

Fig. 1. Our model planet without clouds in $F$ (top) and $|Q|$ (bottom), using MODIS data of the following days (from left to right): July 1, July 28 , August 24, September 20, October 17, November 13, and December 10, 2011, at various sub-observer longitudes and the following phase angles $\alpha: 0^{\circ}, 20.41^{\circ}, 40.82^{\circ}, 60.25^{\circ}, 90.49^{\circ}, 109.92^{\circ}$, and $135.25^{\circ}$. The images are not a temporal sequence, they only illustrate various appearances of the planet. The RGB colors were computed using weighted additive color mixing of the fluxes at $\lambda=443 \mathrm{~nm}$ (blue), $550 \mathrm{~nm}$ (green), and $670 \mathrm{~nm}$ (red), such that when these fluxes are equal, the resulting color is white. The grayscale of each pixel is computed from the sum of the fluxes at the three wavelengths. These images have 220 pixels along the light equator of the planet.

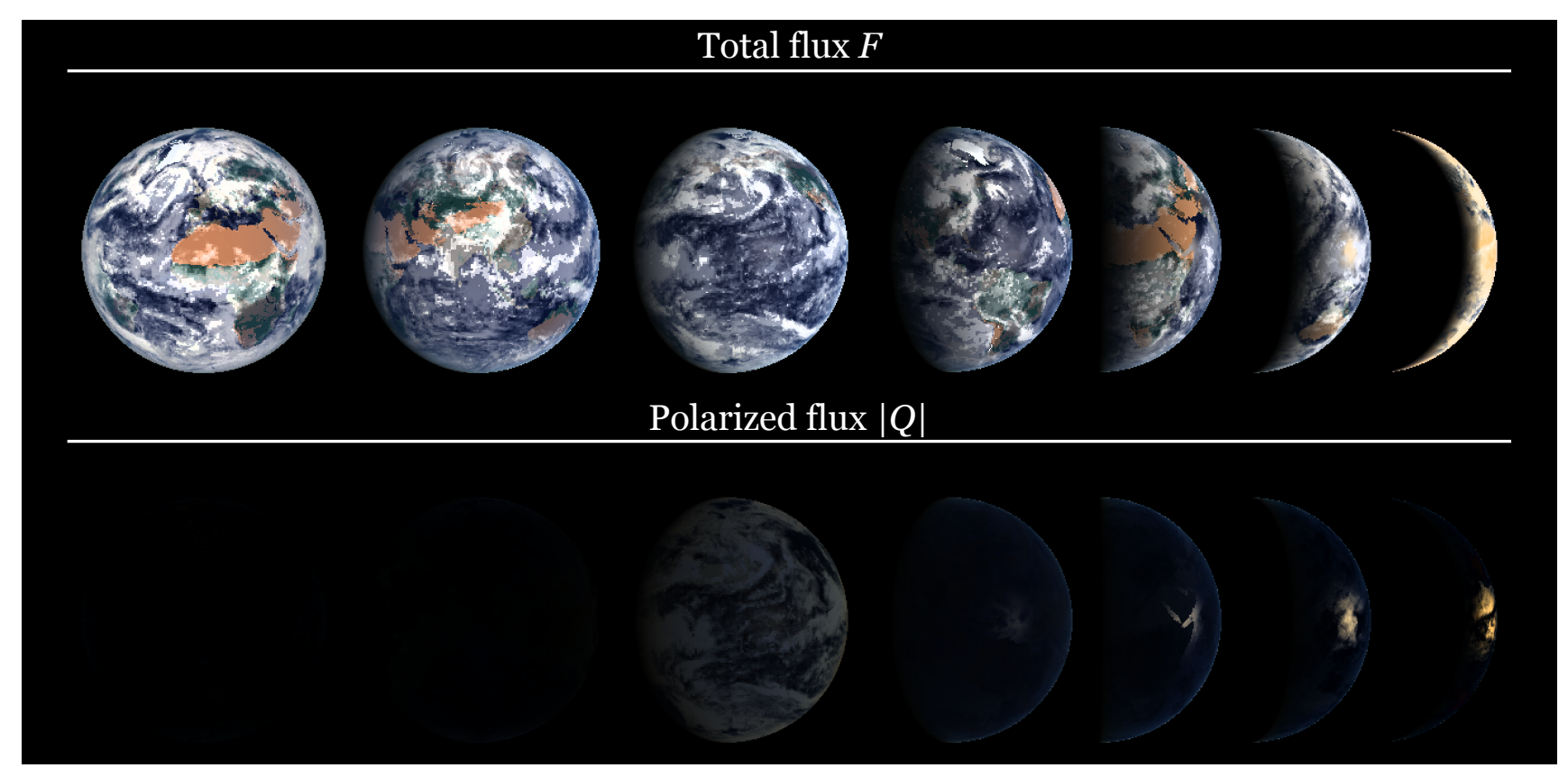

Fig. 2. Similar to Fig. 1, but with cloud properties and patterns from the MODIS data of the respective days.

Fourier series (see de Haan et al. 1987, for details on this expansion) for the various atmosphere-surface combinations on the model planet. We computed and stored the Fourier coefficients at values of $\cos \theta_{0}$ and $\cos \theta$ that coincide with Gaussian abscissae, and additionally at $\cos \theta_{0}=1$ and $\cos \theta=1$. Given local angles $\theta_{0}, \theta$, and $\phi-\phi_{0}$, we can efficiently compute the local $\boldsymbol{R}_{i 1}$ by summing (see de Haan et al. 1987) the Fourier coefficients obtained by interpolating between the relevant stored coefficients.
The Stokes parameters $Q_{i}$ and $U_{i}$ of a locally reflected vector $\boldsymbol{F}_{i}$ are defined with respect to the local meridian plane. To compute the disk-integrated flux vector, we redefined them to the planetary scattering plane before we summed the vectors for all pixels using the rotation matrix $\mathbf{L}_{i}\left(\beta_{i}\right)$, where $\beta_{i}$ is the rotation angle between the two reference planes for a given pixel $i$, see Hovenier \& van der Mee (1983) for the precise definitions of $\mathbf{L}_{i}$ and $\beta_{i}$. We computed the disk-integrated flux vector $\pi \boldsymbol{F}$, as seen 
from an observer at distance $d$, using

$\pi \boldsymbol{F}(\lambda, \alpha)=\frac{F_{0}(\lambda)}{d^{2}} \sum_{i=1}^{N} \mu_{i} \mu_{0 i} \mathbf{L}_{i}\left(\beta_{i}\right) \boldsymbol{R}_{i 1}\left(\lambda, \mu_{i}, \mu_{0 i}, \phi_{i}-\phi_{0 i}\right) d O_{i}$,

where $N$ is the number of pixels, $d O_{i}$ is the 3D surface area covered by pixel $i$, and $\mu_{i}=\cos \theta_{i}$ and $\mu_{0 i}=\cos \theta_{0 i}$ are the cosines of the local angles $\theta_{i}$ and $\theta_{0 i}$, respectively. The surface area $d O_{i}$ is related to the local viewing angle $\theta_{i}$, where $d O_{i} \mu_{i}$ equals the pixel size, which we chose to be the same for all pixels.

We show results at five wavelengths: 350, 443, 550, 670, and $865 \mathrm{~nm}$. To make our results independent of distances, radii, etc., we normalized the reflected flux vectors such that the diskintegrated reflected total flux presented in this paper at $\alpha=0^{\circ}$ equals the planetary geometric albedo (see, e.g., Rossi et al. 2018; Stam 2008), which we denote by " $F$ " in what follows. When Eq. (7) is used, our results can straightforwardly be scaled to a given planetary system and stellar type. Depending on the stellar spectrum, the planetary color in total and polarized flux would change. Because the degree of polarization $P$ is a relative measure (see Eq. (5)), it would not require normalization or scaling.

Furthermore, our results pertain to planets that are spatially resolved from their parent star and background light of the parent star is not included. To translate our results for planets that are spatially unresolved from their star (for examples for closein giant planets, see Seager et al. 2000), the flux vector of the star needs to be added to the planetary flux vector (the starlight is usually unpolarized, Kemp et al. 1987). Spatially resolving a planet in the habitable zone of a solar-type star from its star requires extreme adaptive optics systems and advanced techniques to block the direct starlight. Even with these techniques, background starlight remains at the position of the planet on the detector. However, as it is a differential method, adding polarimetry as a technique enhances the achieved contrast by three to five orders of magnitude (Keller et al. 2010).

We also ignored contributions of starlight scattered by (exo)zodiacal dust, interstellar dust, and instrumental effects to avoid making our results too specific and expanding the parameter space too much. These contributions might be added, similarly to the background starlight. Our model planet has a circular orbit around its star, with an inclination angle of $90^{\circ}$. The obliquity of the planetary rotation axis equals that of Earth, $23.45^{\circ}$, and the rotation period is equal to one sidereal Earth day. The orientation of the planetary rotation axis with respect to the parent star and the observer depends on the day of the year at full phase. The results of our simulations can be adapted for a differently inclined orbit by choosing the appropriate phase angle range, except that the distribution of continents and clouds on the model planet remains the same (e.g. our results do not include a full view of a polar cap of the model planet). By adapting the temporal scale of the results, the rotation period can be adapted, except for the effect that a different rotation period would have on the atmospheric parameters, in particular, on those of the clouds.

\subsection{Computational costs}

The computational costs of our numerical simulations depend on a large number of numerical parameter settings (Rossi et al. 2018; de Haan et al. 1987). For the results presented in this paper, and thus for the data files made publicly available, we chose the most accurate settings without considering the computational costs. In particular, with 100 pixels across the equator of the model planet, about 7850 pixels covered the planetary disk when we modeled the local variations in atmosphere and surface properties (see Sect. 3.1). For every local atmosphere-surface model, we computed the reflected Stokes vector (as coefficients of its expansion in a Fourier series, see Rossi et al. 2018) for up to 151 different local solar zenith angles $\theta_{0}$ and up to 151 different local viewing zenith angles $\theta$. These Fourier-coefficient files were stored in a database for use in the computation of the disk-integrated Stokes vectors. For the latter, we used a temporal resolution for the rotation of the model planet of $15 \mathrm{~min}$, covering 184 Earth days, thus 17664 disk-integrations (with increasing $\alpha$, the number of illuminated pixels on the planetary disk decreases, and the computational costs decrease in turn). With these settings, the computation of a disk-integrated Stokes vector across all phase angles takes about $18 \mathrm{~h}$ on a $2.7 \mathrm{GHz}$ Intel Core i5 processor for a single wavelength.

\section{Atmosphere - surface models}

Here, we describe the local atmosphere and surface properties of the pixels on our model planet. We assign these properties using Earth-observation satellite data.

\subsection{Model atmospheres}

Our model planets have locally plane-parallel layered atmospheres that consist of gaseous molecules and (optionally) cloud particles. We assumed an Earth-like gas mixture in hydrostatic equilibrium (using an Earth-like gravitational acceleration $g$ of $9.81 \mathrm{~m} \mathrm{~s}^{-2}$ ), with a mean molecular mass $m_{\mathrm{g}}$ of $29 \mathrm{~g} \mathrm{~mol}^{-1}$. We only considered continuum wavelengths and thus ignored gaseous absorption. Our shortest wavelength of $350 \mathrm{~nm}$ is just outside the ultraviolet absorption band (the Huggins band) of ozone. For an Earth-like amount and vertical distribution of ozone, the broadband absorption between about 400 and $650 \mathrm{~nm}$ (the Chappuis band) decreases the total reflected flux at $550 \mathrm{~nm}$ wavelength, but it does not significantly affect the degree of polarization at this wavelength, because although in principle, absorption decreases the amount of multiple scattering of light, and thus usually increases the degree of polarization of the reflected light, most of the absorption by ozone takes place at high altitudes in the atmosphere, where the gas density and thus the amount of multiple scattering is small. The strength of absorption bands in reflected light signals depends on the (wavelength-dependent) absorption cross-section of the gas and also strongly depends on the amount and vertical distribution of the ozone molecules, and cloud particles. To avoid introducing too many Earth-specific parameters, we decided to ignore it (for spectra that include absorption by $\mathrm{O}_{3}$ in both the Huggins and the Chappuis bands, see Stam 2008).

The gas molecules are anisotropic Rayleigh scatterers with a wavelength-independent molecular depolarization factor $\delta$ of 0.03 (Hansen \& Travis 1974) and a wavelength-dependent refractive index (Ciddor 1996). The surface pressure $p_{\mathrm{s}}$ is 1.0 bar. Sample values of the atmospheric gaseous (scattering) optical thickness $b_{\mathrm{g}}$ are 0.62 at $\lambda=350 \mathrm{~nm}$ and 0.043 at $670 \mathrm{~nm}$. The atmospheric parameters and their values are given in Table 1.

We divided the atmosphere into three homogeneous layers. The gaseous scattering optical thickness of each layer was computed assuming an exponentially decaying pressure within each layer (for the radiative transfer algorithm, only the optical thickness of the layer is relevant, not the vertical distribution of the gas within the layer). If they were present, the cloud particles were in 
Table 1. Parameters of the model atmosphere.

\begin{tabular}{|c|c|c|}
\hline Parameter and unit & Symbol & Value(s) \\
\hline Surface pressure [bar] & $p_{\mathrm{s}}$ & 1.0 \\
\hline Mean molecular mass $\left[\mathrm{g} \mathrm{mol}^{-1}\right]$ & $m_{\mathrm{g}}$ & 29.0 \\
\hline Molecular depolarization factor [-] & $\delta$ & 0.03 \\
\hline Acceleration of gravity $\left[\mathrm{m} \mathrm{s}^{-2}\right]$ & $g$ & 9.81 \\
\hline Gas optical thickness [-] & $b_{\mathrm{g}}(\lambda)$ & $\begin{array}{l}0.62(350 \mathrm{~nm}) ; \\
0.23(443 \mathrm{~nm}) ; \\
0.10(550 \mathrm{~nm}) ; \\
0.043(670 \mathrm{~nm}) ; \\
0.015(865 \mathrm{~nm})\end{array}$ \\
\hline Cloud particle size distribution & & Two parameter gamma \\
\hline Effective radius $[\mu \mathrm{m}]$ & $r_{\text {eff }}$ & $10 ; 12.5 ; 15 ; 17.5$ \\
\hline Effective variance & $v_{\text {eff }}$ & 0.1 \\
\hline \multicolumn{3}{|l|}{ Cloud particle refractive index } \\
\hline Real part & $n_{\mathrm{r}}$ & 1.33 \\
\hline Imaginary part & $n_{\mathrm{i}}$ & $10^{-8} i$ \\
\hline Cloud optical thickness [-] & $b_{\mathrm{c}}(\lambda)$ & $0 ; 5 ; 10 ; 20$ \\
\hline Cloud top pressure [mbar] & $p_{\mathrm{c}}$ & $500 ; 700 ; 850$ \\
\hline Cloud vertical extent [mbar] & - & 100 \\
\hline
\end{tabular}

the middle layer. The cloud layer always had a vertical extent of $100 \mathrm{mb}$, and the pressure levels of the three layers were adapted to accommodate different cloud top altitudes. Topographical variations were not considered, that is, the bottom of the atmosphere was at 1 bar everywhere, and the top was bounded by space. To limit the computational time and to avoid overcomplicating the models, our model atmospheres do not contain particles other than cloud particles, in other words, we ignored small, suspended, so-called aerosol particles such as dust and/or sea-salt particles, and/or smoke due to biomass burning. Most of these aerosol particles show significant horizontal variability while having relatively small optical thicknesses compared to clouds (for dust, see, e.g., Ginoux et al. 2001). The influence of various types and optical thicknesses of aerosol could be part of a subsequent study.

The spatial and temporal variability in the cloud cover is derived from observations by MODIS, the Moderate Resolution Imaging Spectroradiometer (for a detailed description of the MODIS data sets, including retrieval algorithms, see King et al. 2004; Parkinson \& Greenstone 2000). We describe a cloud by the pressure of its top, $p_{\mathrm{c}}$, its optical thickness, $b_{\mathrm{c}}$, and the cloud particle size distribution and composition.

Each cloudy model pixel has a cloud fraction. This is zero for a cloud-free pixel. Even with as many as 100 pixels along the light equator of the model planet, the pixels are much larger than typical horizontal variations in clouds, and they are much larger than MODIS ground pixels. For a mixture of cloudfree and partly and fully cloudy MODIS ground pixels within a model pixel, we computed the locally reflected Stokes vector for a cloud-free and a cloudy model pixel, and took their weighted sum to compute the reflected Stokes vector for the pixel as a whole. In this way, we accounted for the cloud fraction as reported for all cloudy MODIS ground pixels. The mean cloud fraction of our model planet is about $66 \%$.

The MODIS database provides a cloud top pressure for all of its pixels, and we computed $p_{\mathrm{c}}$ of a cloudy model pixel by taking the average of the cloud top pressures of the MODIS pixels within our model pixel, weighted by their cloud fractions. To limit the number of different atmosphere-surface models, we divided $p_{\mathrm{c}}$ for the model pixels into three categories: $500 \mathrm{mbar}$ for $0 \leq p_{\mathrm{c}}<600 \mathrm{mbar}, 700 \mathrm{mbar}$ for $600 \leq p_{\mathrm{c}}<800 \mathrm{mbar}$, and 850 mbar for $p_{\mathrm{c}} \geq 800 \mathrm{mbar}$ (see Table 1).

We computed $b_{\mathrm{c}}$ for a cloudy model pixel by taking the mean of the cloud optical thickness values of the MODIS ground pixels within the model pixel. In particular, we take the mean of the MODIS logarithmic daily mean data set (see Table 2). This data set provides the cloud optical thickness for a given MODIS pixel $i$ as $\log b_{\mathrm{c} i}$, where $b_{\mathrm{c} i}$ is the cloud optical thickness. When we computed the mean $b_{\mathrm{c}}$, we weighted $\log b_{\mathrm{c} i}$ of each MODIS pixel with its cloudiness fraction. This logarithmic mean as a good approximation for the cloud optical thickness has been proposed by Hubanks et al. (2015) and Oreopoulos et al. (2007).

The wavelength at which a cloud optical thickness is provided in the MODIS data set depends on the surface: $b_{\mathrm{c}}$ pertains to $645 \mathrm{~nm}$ above a dry surface, to $858 \mathrm{~nm}$ above an ocean, and to $1240 \mathrm{~nm}$ above snow or ice. We obtain $b_{\mathrm{c}}$ at another wavelength by multiplying the value from the data set with the ratio of the cloud particle extinction cross-section at that other wavelength and at the given wavelength. The cloud optical thickness depends somewhat on the wavelength: for a cloud with $b_{\mathrm{c}}=10$, composed of particles with $r_{\text {eff }}=12.5 \mu \mathrm{m}$ and $v_{\text {eff }}=0.1$, the cloud optical thickness, for example, ranges from 9.775 to 10.002 , from 350 to $865 \mathrm{~nm}$. The extinction cross-sections of the cloud particles were computed using a Mie-algorithm based on De Rooij \& van der Stap (1984). To limit the number of different atmospheresurface models, we divided $b_{\mathrm{c}}$ for the model pixels into four categories: 0.0 when $b_{\mathrm{c}}<0.01,5.0$ when $0.01 \leq b_{\mathrm{c}}<7.5,10.0$ when $7.5 \leq b_{\mathrm{c}}<15.0$, and 20 when $b_{\mathrm{c}}>15$, at 645,858 , or $1240 \mathrm{~nm}$, depending on the surface (see above).

The cloud particles were assumed to be homogeneous, liquid-water spheres, with a refractive index of $n=1.33+10^{-8} i$ (Hale \& Querry 1973). Their sizes are described by a twoparameter gamma distribution (Hansen \& Travis 1974), with an effective particle radius $r_{\text {eff }}$ and an effective variance $v_{\text {eff }}$. We used $v_{\text {eff }}=0.1$ (Han et al. 1994; Nakajima \& King 1990). The cloud particle effective radius was provided by the MODIS database, and we computed $r_{\text {eff }}$ for a model pixel by taking the average of the effective radii of the MODIS pixels within our model pixel, weighted with the cloud fractions. We computed the single-scattering properties (i.e. single-scattering matrix, albedo, and extinction cross-section) of the cloud particles using a Mie algorithm based on De Rooij \& van der Stap (1984).

To limit the number of different atmosphere-surface models, we divided $r_{\text {eff }}$ for the model pixels into four categories (see Nakajima \& King 1990): $10 \mu \mathrm{m}$ for $0.0 \leq r_{\text {eff }} \leq 11.25 \mu \mathrm{m}$, $12.4 \mu \mathrm{m}$ for $11.25<r_{\mathrm{eff}} \leq 13.75 \mu \mathrm{m} 15.0 \mu \mathrm{m}$ for $13.75<r_{\mathrm{eff}} \leq$ $16.25 \mu \mathrm{m}$, and $17.5 \mu \mathrm{m}$ for $r_{\mathrm{eff}}>16.25 \mu \mathrm{m}$.

\subsection{Model surfaces}

The surface of our model Earth was covered by four surface types: ocean, vegetation, soil, and snow or ice. Vegetation was subdivided into deciduous forest, grass, and steppe. Soil was subdivided into sand desert and shrubland. For the spatial and temporal variability in surface type we again used MODIS data. When the MODIS data showed various surface types within a model pixel, we assigned the most abundant surface type to the pixel. The different surface types and their reflection properties are described in more detail below.

\subsubsection{Ocean}

The ocean consisted of a Fresnel-reflecting and transmitting airwater interface with water below. The interface was rough due 
Table 2. References of the parameter databases (upper part of the table) and of the surface models (lower part of the table) used for the Earth-like model planet.

\begin{tabular}{|c|c|c|}
\hline$\underline{\text { Parameter }}$ & Reference & Comments \\
\hline Cloud optical thickness & Platnick et al. (2015) & $\begin{array}{l}\text { Satellites: Aqua and Terra. Instrument: MODIS. } \\
\text { Logarithmic daily mean data set (see Hubanks et al. 2015; } \\
\text { Oreopoulos et al. 2007). } \\
\text { Data range: January } 1,2011 \text { - December } 31,2011 \text {. } \\
\text { Temporal resolution: one day. } \\
\text { Spatial resolution: } 1.0^{\circ} \times 1.0^{\circ} \text {. }\end{array}$ \\
\hline Cloud particle effective radii & Platnick et al. (2015) & $\begin{array}{l}\text { Satellites: Aqua and Terra. Instrument: MODIS. Daily mean data set. } \\
\text { Data range: January } 1,2011 \text { - December } 31,2011 \text {. } \\
\text { Temporal resolution: one day. } \\
\text { Spatial resolution: } 1.0^{\circ} \times 1.0^{\circ} \text {. }\end{array}$ \\
\hline Cloud top pressure & Platnick et al. (2015) & $\begin{array}{l}\text { Satellites: Aqua and Terra. Instrument: MODIS. Daily mean data set. } \\
\text { Data range: January } 1,2011 \text { - December } 31,2011 \text {. } \\
\text { Temporal resolution: one day. } \\
\text { Spatial resolution: } 1.0^{\circ} \times 1.0^{\circ} \text {. }\end{array}$ \\
\hline Surface cover type & Friedl \& Sulla-Menashe (2019) & $\begin{array}{l}\text { Satellites: Combined Aqua and Terra. Instrument: MODIS. } \\
\text { Data range: January } 1,2011 \text { - December } 31,2011 \text {. } \\
\text { Temporal resolution: Yearly. } \\
\text { Spatial resolution: } 0.05^{\circ} \times 0.05^{\circ} \text {. }\end{array}$ \\
\hline Snow cover & Hall \& Riggs (2016) & $\begin{array}{l}\text { Satellite: Aqua. Instrument: MODIS. } \\
\text { Data range: January } 1,2011 \text { - December } 31,2011 \text {. } \\
\text { Temporal resolution: Daily. } \\
\text { Spatial resolution: } 0.05^{\circ} \times 0.05^{\circ} \text {. }\end{array}$ \\
\hline Wind speed above the ocean & Wentz et al. (2015); Atlas et al. (2011) & $\begin{array}{l}\text { The input data are a combination of intercalibrated satellite data from } \\
\text { numerous radiometers and scatterometers and in situ data from moored } \\
\text { buoys. } \\
\text { Data range: January } 1,2011 \text { - December } 31,2011 \text {. } \\
\text { Wind speed altitude: } 10 \text { meters. Temporal resolution: } 6 \mathrm{~h} \text {. } \\
\text { Spatial resolution: } 0.25^{\circ} \times 0.25^{\circ} \text {, near globally. }\end{array}$ \\
\hline Pale soil albedo & Tilstra et al. (2017) & $\begin{array}{l}\text { Satellite: ENVISAT. Instrument: SCIAMACHY. } \\
\text { Lambertian-equivalent reflectivity data set. } \\
\text { Temporal resolution and coverage: one month in } 2002-2012 \text {. } \\
\text { Spatial resolution: } 1.0^{\circ} \times 1.0^{\circ} \text {. } \\
\text { Soil albedo based on the mean spectral reflectivity over a full year } \\
\text { and over a slab of desert sands in Egypt, with the coordinates } \\
26.25^{\circ}-27.25^{\circ} \text { latitude, } 26.25^{\circ}-27.25^{\circ} \text { longitude. }\end{array}$ \\
\hline Dark brown soil albedo & Meerdink et al. (2019); Baldridge et al. (2009) & $\begin{array}{l}\text { Retrieved from https://speclib.jpl.nasa.gov } \\
\text { Library developed as part of the ASTER and ECOSTRESS projects. }\end{array}$ \\
\hline Ice and snow albedo & Meerdink et al. (2019); Baldridge et al. (2009) & Same comment as provided for dark brown soil albedo. \\
\hline Deciduous forest albedo & Meerdink et al. (2019); Baldridge et al. (2009) & Same comment as provided for dark brown soil albedo. \\
\hline Grass albedo & Meerdink et al. (2019); Baldridge et al. (2009) & Same comment as provided for dark brown soil albedo. \\
\hline Steppe albedo & ARM-facility (1998) & $\begin{array}{l}\text { Spectral albedo retrieved from a patch of ground in the Southern } \\
\text { Great Planes, USA, using a multifilter radiometer at } 25 \text { m altitude. } \\
\text { The patch of ground consisted of } 56 \% \text { vegetation, the remaining } \\
\text { part was soil. The measurement was taken on June } 20,2011 \text {. }\end{array}$ \\
\hline Scattering coefficients of soil & Moreno et al. (2006) & $\begin{array}{l}\text { Retrieved from the Amsterdam light-scattering } \\
\text { database (Muñoz et al. 2012). }\end{array}$ \\
\hline Surface model & Reference & Comments \\
\hline Ocean & Trees \& Stam (2019) & $\begin{array}{l}\text { Rough Fresnel-reflecting interface, subinterface pure seawater body, } \\
\text { ocean bottom, and wind-generated foam. }\end{array}$ \\
\hline Deciduous forest & Maignan et al. (2009); Roujean et al. (1992) & $\begin{array}{l}\text { Combined vegetation model, which includes the complete reflection } \\
\text { matrix for the total reflection and linearly polarized reflection. } \\
\text { Circularly polarized reflection not included. }\end{array}$ \\
\hline Grass & Maignan et al. (2009); Roujean et al. (1992) & $\begin{array}{l}\text { Combined vegetation model, which includes the complete reflection } \\
\text { matrix for the total reflection and linearly polarized reflection. } \\
\text { Circularly polarized reflection not included. }\end{array}$ \\
\hline Steppe & Maignan et al. (2009); Roujean et al. (1992) & $\begin{array}{l}\text { Combined vegetation model, which includes the complete reflection } \\
\text { matrix for the total reflection and linearly polarized reflection. } \\
\text { Circularly polarized reflection not included. }\end{array}$ \\
\hline Desert & $\begin{array}{l}\text { Amsterdam light-scattering database } \\
\text { (Muñoz et al. 2012) }\end{array}$ & $\begin{array}{l}\text { Pale soil. Optically thick dust layer }(b=100) \text { just above a black surface. } \\
\text { Mie scattering matrix provided at } 663 \mathrm{~nm} \text {. See text for details. }\end{array}$ \\
\hline Shrublands & $\begin{array}{l}\text { Amsterdam light-scattering database } \\
\text { (Muñoz et al. 2012) }\end{array}$ & $\begin{array}{l}\text { dark brown soil. Reflection model the same as that of the desert, } \\
\text { but scaled for the albedo of dark brown soil. See text for details. }\end{array}$ \\
\hline Ice or snow & - & Lambertian-reflecting surface with appropriate ice or snow albedo. \\
\hline
\end{tabular}


Table 3. Parameters of the ocean.

\begin{tabular}{lll}
\hline \hline Parameter and unit & Symbol & Value \\
\hline Wind speed $\left[\mathrm{m} \mathrm{s}^{-1}\right]$ & $v$ & $5.0 ; 7.0$ \\
Foam albedo & $a_{\text {foam }}$ & 0.22 \\
Depolarization factor water & $\delta_{\text {water }}$ & 0.09 \\
Refractive index above the air-water interface & $n_{1}$ & 1.0 \\
Refractive index below the air-water interface & $n_{2}$ & 1.33 \\
Chlorophyll concentration [mg m & -3 \\
Ocean depth [m] & {$[$ Chl] } & 0.0 \\
Ocean bottom surface albedo & $\Delta z_{\text {ocean }}$ & 100.0 \\
\hline
\end{tabular}

to wind-driven waves that were described by randomly oriented flat facets. The standard deviation of the wave-facet inclinations increased with the wind speed, widening the sun-glint pattern on the surface (Mishchenko \& Travis 1997; Cox \& Munk 1954). We included scattering of light within the water body by describing it as a stack of horizontally homogeneous layers of pure seawater and by computing its reflection using an adding-doubling algorithm (de Haan et al. 1987). The sub-ocean surface was black.

The single scattering in pure seawater is described by anisotropic Rayleigh scattering (Hansen \& Travis 1974) with a depolarization factor of 0.09 (Chowdhary et al. 2006; Morel 1974), combined with realistic absorption and scattering coefficients for pure seawater (Sogandares \& Fry 1997; Pope \& Fry 1997; Smith \& Baker 1981). This results in a natural blue (for low chlorophyll concentrations) water body. The real part of the refractive index of the water was assumed to be 1.33 (Hale \& Querry 1973) and wavelength independent, which does not significantly influence the ocean reflection. Furthermore, we took the reflection by wind-generated surface foam into account (see Koepke 1984) and corrected for the energy surplus across the rough air-water interface at grazing angles that was caused by neglecting wave shadowing (see also Zhai et al. 2010; Tsang et al. 1985).

To limit the number of different atmosphere-surface models, we divided the ocean surface wind speed $v$ for the model pixels into two categories: $5 \mathrm{~m} \mathrm{~s}^{-1}$ for $v<6 \mathrm{~m} \mathrm{~s}^{-1}$, and $7 \mathrm{~m} \mathrm{~s}^{-1}$ for $v \geq 6 \mathrm{~m} \mathrm{~s}^{-1}$. This division is based on the global variation of mean wind speed measurements between January 1, 2011, and December 31, 2011, at an altitude of 10 meters (see Atlas et al. 2011) ${ }^{3}$. The parameters of the ocean properties are listed in Table 3. For a detailed description of our ocean reflection algorithm, see Trees \& Stam (2019).

\subsubsection{Vegetation}

A vegetated surface on our model planet can be covered by deciduous forest, grass, or steppe. We used a combination of surface reflection models to describe the bidirectionally reflected total and linearly polarized fluxes. We also accounted for the reflection of incident linearly polarized light, as the diffuse skylight is usually linearly polarized. We ignored the reflection of incident circularly polarized light, however, as this signal is very weak (Rossi \& Stam 2018). We also ignored the circular polarization of light that is reflected by vegetation. This light is expected to be slightly circularly polarized (Patty et al. 2019; Sparks et al. 2009), but to our knowledge, there is no numerical

3 Cross-calibrated multi-platform (CCMP) wind vector analysis, http://www . remss . com/measurements/ccmp/ bidirectional reflection model for vegetation that includes circular polarization (the measurements by Patty et al. 2019 cover a range of incidence and reflection angles that is, as yet too limited to convert the data into a fully bidirectional reflection model).

To describe the bidirectionally reflected total flux, we used the numerical model developed by Roujean et al. (1992),

$$
\begin{aligned}
\rho\left(\theta, \theta_{0}, \phi-\phi_{0}, \lambda\right)= & k_{0}(\lambda)+k_{1}(\lambda) f_{1}\left(\theta, \theta_{0}, \phi-\phi_{0}\right) \\
& +k_{2}(\lambda) f_{2}\left(\theta, \theta_{0}, \phi-\phi_{0}\right),
\end{aligned}
$$

where $\rho$ is the bidirectional reflectance. The function $f_{1}$ is the so-called geometric component of the reflection, which includes the influence of shadows (mutual shadows, such as one tree casting a shadow on another tree, are ignored), and $f_{2}$ is the volume component, which includes the amount of local reflection (e.g. the density of leaves in a tree canopy) (for details, see Roujean et al. 1992). Functions $f_{1}$ and $f_{2}$ vanish at $\theta=\theta_{0}=0^{\circ}$. Using remote-sensing data, Roujean et al. (1992) derived parameters $k_{0}, k_{1}$, and $k_{2}$ for various types of vegetation and two wavelength bands: 580 to $680 \mathrm{~nm}$ and 730 to $1100 \mathrm{~nm}$.

To account for the spectral variability of the reflection within these bands, in particular the green bump and red-edge features, the $k$-parameters were scaled to the appropriate hemispherical albedo of the vegetation type considered. The hemispherical albedo of deciduous forest and grasslands was taken from the ECOSTRESS spectral library (Meerdink et al. 2019; Baldridge et al. 2009), and that of steppe from the Atmospheric Radiation Measurement research facility (ARM-facility 1998) (see Table 2).

To account for the reflected linearly polarized fluxes, we used a modified version of the numerical model developed by Maignan et al. (2009), following Schaepman-Strub et al. (2006), to obtain a bidirectional polarization distribution function (BPDF). This BPDF is a linear one-parameter-model, a simplification of the nonlinear, two-parameter model by Nadal $\&$ Breon (1999). To account for different types of vegetation, the BPDF uses the normalized difference vegetation index (NDVI) and a so-called $\alpha$-parameter. A table with these parameters for several types of vegetation is provided in Maignan et al. (2009). With the NDVI parameter, the model of Maignan et al. (2009) accounts for the spectral variation in total flux, and with the $\alpha$ parameter, a best fit with observations of the vegetation type considered is achieved (see Maignan et al. 2009, for a detailed description).

\subsubsection{Arid regions}

We used two types of arid regions: sandy deserts, and shrublands. For these surface types, we assumed the same reflection functions, but different spectral albedos. We approximated the slightly polarized bidirectional reflection using the reflection matrix of an optically thick (i.e. $b_{\text {ext }}=100$ ) layer of irregularly shaped mineral particles. This matrix was computed using our adding-doubling algorithm. For the single-scattering matrix of the mineral particles, we used that of the olivine $\mathrm{S}$ particles (Muñoz et al. 2012; Moreno et al. 2006), while we scaled the single-scattering albedo of the mineral particles to fit the hemispherical albedo of pale soil for the sandy desert, and dark brown entisol for the shrubland. The spectral albedos and databases we used are provided in Table 2.

\subsubsection{Ice or snow cover}

We described fields of snow and ice on land and inland waters as Lambertian reflectors. Their reflection is thus isotropic and 
unpolarized. A flat, smooth ice sheet free of rocks, snow or other rough materials might also be approximated as a Fresnel reflector. However, given the physical dimensions of our model pixels, and thus their spatial extent on the surface, a Lambertian approximation appears to be fitting well (Coakley 2003). To account for temporal changes in snow-covered land and inland water, we applied the daily global snow cover mask of MODIS on the yearly surface cover type data set. We did not account for sea ice. The names of the spectral albedo and surface type databases are provided in Table 2 .

\section{Results}

\subsection{RGB-images}

Figures 1 and 2 show RGB images of our model Earth without and with clouds at various orientations and phase angles. These figures illustrate the variation in RGB colors of the total and polarized fluxes $F$ and $|Q|$ across the model planet. The model parameters are as described in Sect. 3. The RGB images clearly show that the disk-integrated fluxes are expected to vary spectrally and temporally as the planet rotates and orbits its star. A particularly striking feature on the cloud-free disks is the glint, that is, the sunlight that is reflected by the ocean, the contribution of which to the signal of the disk increases with increasing phase angle $\alpha$. The glint can also be seen on the cloudy disks, but it is more diffuse, as clouds hide the ocean and its reflection from view. The reddening of the glint in $F$ and $|Q|$ with increasing $\alpha$ has been described by Trees \& Stam (2019). These authors also argued that the reddening in $|Q|$ is indicative of the presence of a cloud-free or cloudy ocean, while $F$ also reddens when the planet has no ocean, but only clouds. The slight increase in $|Q|$ of the cloudy model planet at $\alpha=40^{\circ}$ is due to the primary rainbow, that is, light that has been reflected inside the cloud droplets once (see, e.g., Karalidi et al. 2012b; Stam 2008; Bailey 2007).

\subsection{Phase curves}

Figure 3 shows $F, Q, U, V$, and the degree of polarization $P$ of light reflected by the rotating cloud-free model planet as functions of $\alpha$, and for $\lambda$ ranging from 350 to $865 \mathrm{~nm}$. The simulations start at $\alpha=0^{\circ}$ on July 1, 12:00 GMT, 2011 (the geographic longitude centered at the illuminated and visible disk is $0^{\circ}$, like in Figs. 1-2), and stop at $\alpha=180^{\circ}$ on December 31, 11:45 GMT, 2011. The figure also includes curves for a cloudfree model planet whose surface is completely covered by ocean (the "ocean planet" phase curves of Fig. 1 of Trees \& Stam 2019) with a surface wind speed $v$ of $7 \mathrm{~m} \mathrm{~s}^{-1}$, instead of by a mixture of oceans and continents. Figure 4 is similar to Fig. 3, but has clouds. In Figs. 3 and 4, the thin lines were computed with a temporal resolution of $15 \mathrm{~min}$, while the thicker, smoother lines are the averages over $24 \mathrm{~h}$ ( $\alpha$ increases by about $1^{\circ}$ in this period).

The two figures show that $|U|$ is about a factor 100 smaller than $|Q|$ across most of the phase angle range because our reference plane coincides with the light equator of the planet, and therefore any polarized flux $|U|$ is due to secondary and higherorder scattered light, which has a low degree of polarization when integrated across the disk, even in the presence of horizontal inhomogeneities. The polarized flux $|U|$ is highest at the shortest wavelengths because because there, the atmospheric gaseous optical thickness is highest there and thus more multiple scattering takes place. This is especially apparent for the cloudy model planet, where multiple scattering in the gaseous atmosphere above the clouds produces much more $|U|$ at $350 \mathrm{~nm}$ than at $443 \mathrm{~nm}$, where the gas optical thickness above the clouds is far lower.

The circularly polarized flux $|V|$ is a factor of about $10^{4}$ lower than $Q$. This flux only arises for light that has been scattered at least twice, including at least once by the cloud particles, as Rayleigh scattering alone does not produce circular polarized fluxes (see Rossi \& Stam 2018, and references therein). When a model planet is mirror-symmetric with respect to the planetary scattering plane, the circularly polarized flux of the hemisphere above the scattering plane (the northern hemisphere) will be equal to that of the hemisphere below the scattering plane (the southern hemisphere), except for the sign: because of the mirror symmetry in the scattering geometries, $V$ of the northern hemisphere equals $-V$ of the southern hemisphere (see García Muñoz 2015 , for comparable phase curves of $V / I$ as computed for the two hemispheres of the Earth, except with a black surface to model the ocean, while our ocean is modelled as a rough, Fresnel reflecting surface with scattering in the water body below). Integrated over the planetary disk, $V$ of a mirror-symmetric planet will thus equal zero. For a horizontally inhomogeneous planet, such as our model planets, the disk-integrated $|V|$ is usually not zero, but it is still very small, and it decreases with $\lambda$ as the atmosphere's Rayleigh scattering optical thickness decreases with the wavelength. The circular polarization signature of light scattered by chiral molecules, such as those found e.g. in the cells making up Earth-like vegetation, on the northern hemisphere will have the same sign as that on the southern hemisphere, and will thus not be cancelled when integrated across the planetary disk, even though the total signal will still be small because the fraction of circularly polarized flux that is reflected by chiral molecules is very small (see Patty et al. 2019).

Figures 3 and 4 show that except at $\alpha>120^{\circ}$, the average $(24 \mathrm{~h})$ total and polarized fluxes decrease with increasing $\lambda$ below $670 \mathrm{~nm}$. The reason is that the Rayleigh scattering optical thickness in the atmosphere decreases with $\lambda$ (our model atmospheres contain no ozone, otherwise the fluxes at $350 \mathrm{~nm}$ would have been much lower than shown here), and because the dark ocean suppresses the terrestrial average surface albedo. The total flux $F$ at $865 \mathrm{~nm}$ is an exception: it is higher than at $670 \mathrm{~nm}$ (for $\alpha<120^{\circ}$ ). The reason is that at $865 \mathrm{~nm}$, the albedo of vegetation is higher than at $670 \mathrm{~nm}$. The increase in this albedo with increasing $\lambda$ above about $670 \mathrm{~nm}$ is usually referred to as the red edge (Montañés-Rodríguez et al. 2006; Seager et al. 2005). Because of the low-polarization signature of the vegetation, $|Q|$ at $865 \mathrm{~nm}$ is lower than at $670 \mathrm{~nm}$.

For $\alpha>120^{\circ}$, the average $(24 \mathrm{~h})$ cloud-free model planet changes color from blue through white to red because of the presence of the Fresnel-reflecting ocean (see Fig. 1 in Trees \& Stam 2019). The contribution of the ocean glint (which itself is spectrally neutral) increases with increasing $\lambda$ as the Rayleigh scattering optical thickness in the atmosphere decreases. Trees \& Stam (2019) showed that in $F$, this color change may also result from Rayleigh-scattering gas above the clouds, that is, also in the absence of an ocean. The diurnal average of $P$ changes color at $\alpha \sim 144^{\circ}$ and the diurnal average of $Q$ changes color at $\alpha \sim 138^{\circ}$. Trees \& Stam (2019) postulated that the color change of $P$ from blue through white to red with increasing $\alpha$ identifies a partly cloudy exo-ocean, while the color change in $Q$ uniquely identifies an exo-ocean, independent of the surface pressure or cloud fraction.

Compared with the results in Trees \& Stam (2019), where all model planets were ocean planets, Figs. 3 and 4 show that when the model planet has oceans and continents, $P$ and $Q$ still change color, and their color change is thus still indicative of the 

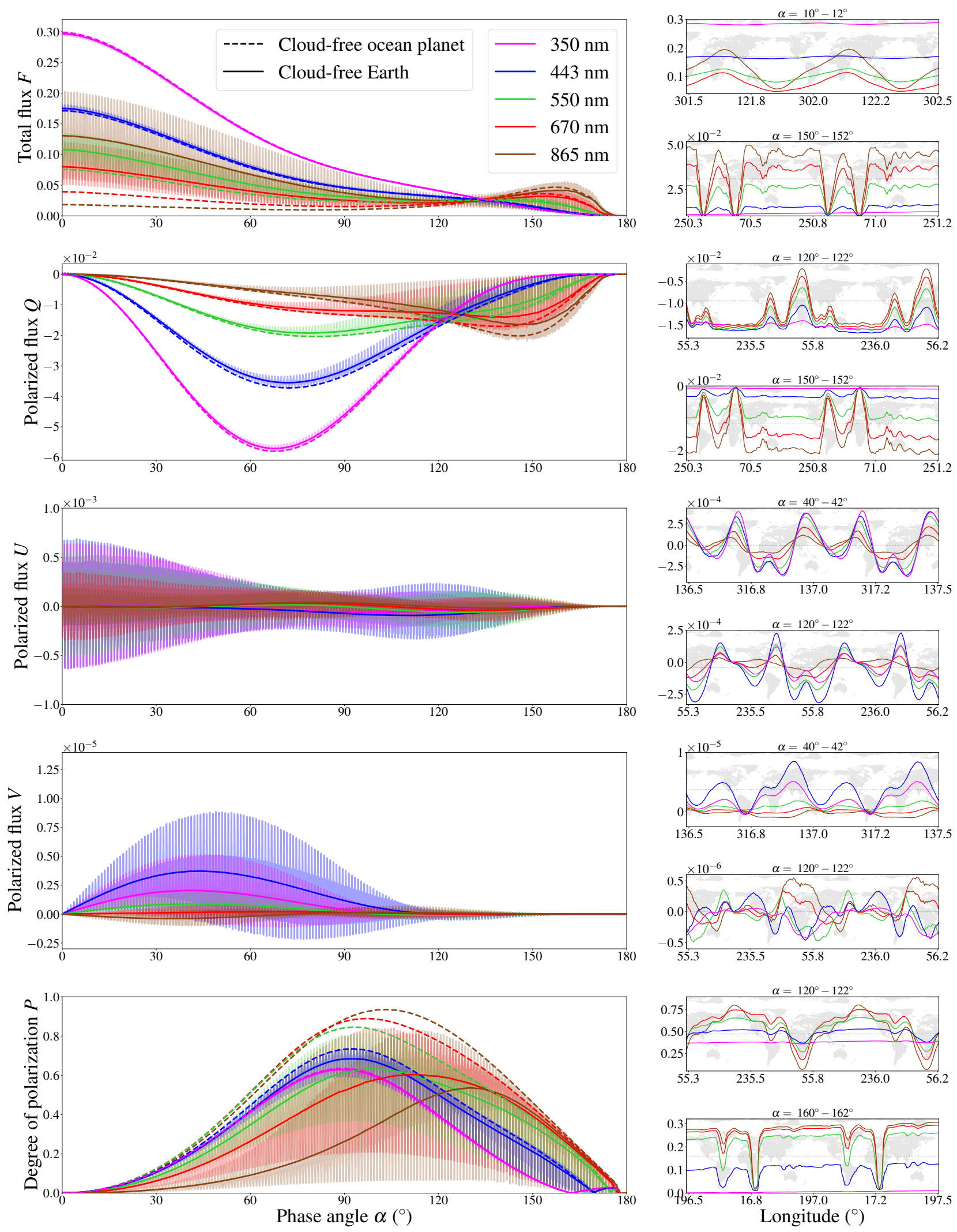

Fig. 3. Numerical results for the cloud-free model Earth. Left: total flux $F$, linearly polarized fluxes $Q$ and $U$, circularly polarized flux $V$, and degree of polarization $P$ as functions of $\alpha$ at $\lambda=350 \mathrm{~nm}$ (pink lines), $443 \mathrm{~nm}$ (blue), $550 \mathrm{~nm}$ (green), $670 \mathrm{~nm}$ (red), and $865 \mathrm{~nm}$ (brown). The temporal resolution of the thin lines is $15 \mathrm{~min}$. The thick solid lines are the diurnal $(24 \mathrm{~h})$ averages. At $\alpha=0^{\circ}$, the time is July $1,12: 00 \mathrm{GMT}$, 2011. At $\alpha=180^{\circ}$, the time is December 31, 11:45 GMT, 2011. The thick dashed lines are the diurnal averages of a cloud-free "ocean planet" (cf. Fig. 1 of Trees \& Stam 2019), thus of a planet without clouds and continents. Right: close-ups of the 15-min time-resolution phase curves for two phase-angle regions as functions of the longitude in the middle of the longitude range that is visible to the observer. The background of each close-up shows the corresponding geographic map of Earth. Note the different vertical scales. 
A. Groot et al.: Colors of an Earth-like exoplanet
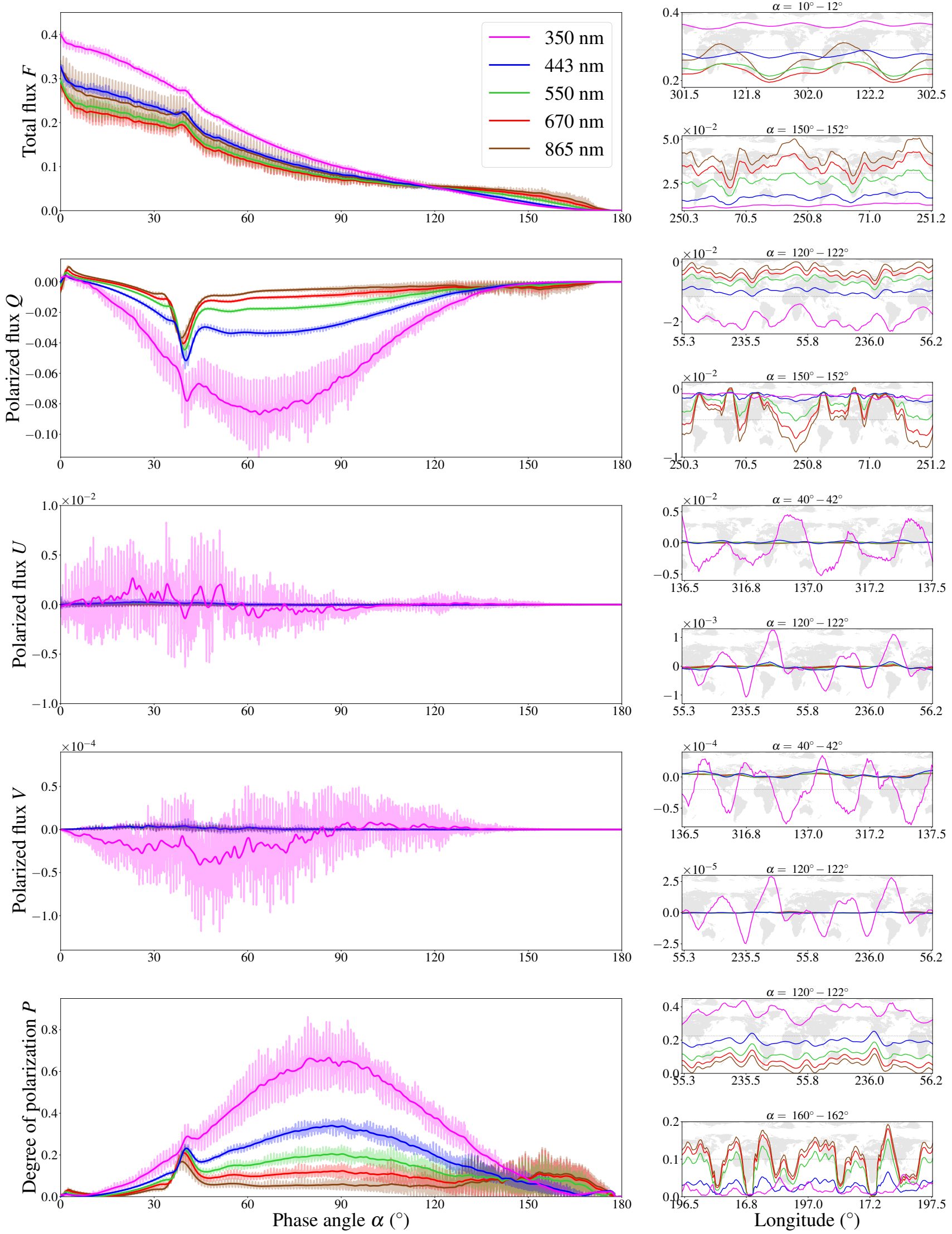

Fig. 4. Similar to Fig. 3 but with clouds. 
presence of an ocean. On Earth, $71 \%$ of the surface is covered by ocean, and in the equatorial regions where the glint occurs, the coverage is even higher.

The average (24h) $F, Q$, and $P$ phase curves of the cloudy planet (Fig. 4) show the familiar features that are due to the scattering of light by the cloud particles, in particular the primary rainbow near $\alpha=40^{\circ}$ that is due to light that has been reflected once within the spherical liquid-water droplets (Karalidi et al. 2011; Stam 2008; Bailey 2007), an enhancement that was also identified in Fig. 2. Fluxes $U$ and $V$ also appear to have a feature at the primary rainbow angle, although not very obvious. At phase angles below $5^{\circ}$, the curves show a small enhancement that is due to the glory formed by light that is back-scattered by the spherical cloud droplets (see, e.g., Hansen \& Travis 1974; Bryant $\&$ Jarmie 1974). At such small phase angles, an exoplanet would be extremely close to its star.

The close-ups of the diurnal variations (the 15 min curves) in Fig. 3 show patterns that repeat about every $1^{\circ}$ in $\alpha$ and are caused by continents and oceans that rotate in and out of view. In Fig. 4 the clouds add variation to the modulation through the surface features and thus suppress the repeated patterns. The temporal variation in $F, Q$, and $P$ increases with increasing $\alpha$ because with decreasing illuminated area on the planetary disk, local variations in surface and cloud coverage contribute more prominently to the reflected signal, as also described by Stam (2008), for example. With increasing $\alpha$, the contribution of the ocean glint to $F$ and $Q$ also increases (cf. Fig. 2). At short wavelengths, the peak-to-peak variability in $F$ and $Q$ of the cloud-free planet (Fig. 3) is small because the Rayleigh scattering optical thickness of the atmosphere is high and suppresses the surface signals. With increasing $\lambda$, the Rayleigh scattering decreases and the peak-to-peak variations due to surface albedo variations are increasingly visible.

In the presence of clouds (Fig. 4), $Q$ and the very low fluxes $U$ and $V$ show the strongest peak-to-peak variability at the shortest wavelength $(350 \mathrm{~nm})$. The temporal variations in $U$ and $V$ appear to be inversely correlated at small $\alpha$ and correlated at larger $\alpha$, which can probably be explained by the fact that both $U$ and $V$ are due to multiple scattered light. The precise relation requires further investigations, however. This strong variability is correlated with the variability of the clouds: without clouds, the polarized fluxes are due to incident sunlight that is scattered upward by the atmospheric gases. Clouds strongly increase the total flux that is reflected by a pixel, and while $P$ of the flux reflected by the cloud is usually low, part of this reflected light is scattered in the gas above the cloud before it escapes into space. Because this last scattering is in the gas, it significantly adds in particular to the planetary linearly polarized flux $Q$. With increasing $\lambda$, the amount of light that is scattered in the gas above the cloud decreases, and with this, the contribution to the polarized fluxes and the sensitivity to the cloud variability decrease.

Robinson et al. (2011) and Fujii et al. (2011) studied the diurnal variability in Earth's reflected total flux $F$ and compared results from their numerical models to reflected total-flux observations of the Earth taken by the EPOXI mission (on three separate days in 2008 , at $\alpha=57.7^{\circ}, 75.1^{\circ}$, and $76.6^{\circ}$, respectively, although Fujii et al. 2011 did not use the $75.1^{\circ}$ data because the moon was partially in view), while Jiang et al. (2018) analyzed reflected total-flux data of the DSCOVR mission (taken only at $\alpha=0^{\circ}$ ). García Muñoz (2015) used his radiative transfer code to simulate 2005 MESSENGER data (reflected total fluxes) between $\alpha=98^{\circ}$ to $107^{\circ}$. While quantitative comparisons between our computed reflected total fluxes and the measured data and computed fluxes presented in these papers is difficult because of differences in cloud coverage and orientation of the model planet (when limited phase angles are covered in the observations), and because different definitions were used, for instance (e.g., fluxes are shown as normalized to a $24 \mathrm{~h}$ time average). We can, however, perform a qualitative comparison (only for the total fluxes because polarized fluxes of the Earth as a whole, have not been measured from space).

In general, Robinson et al. (2011) and Fujii et al. (2011) reported a similar increase in the peak-to-peak variability of the reflected $F$ data with increasing $\lambda$ as we, and the wavelength dependence of $F$ is similar (across the phase-angle range of the EPOXI data); the Earth is darkest around $650 \mathrm{~nm}$ (Fujii et al. 2011) or $670 \mathrm{~nm}$ (our simulations). Jiang et al. (2018) showed a stronger peak-to-peak variability in $F$ at $780 \mathrm{~nm}$ than at $380 \mathrm{~nm}$ at the $0^{\circ}$ phase angle of the DSCOVR data (the spectral solar flux was included in the reflected flux data in their paper). Our diurnal curves as shown in Fig. 4 (diurnal curves near fullphase) agree (qualitatively) well with DSCOVR observations, with strong enhancements in $F$ when large convective clouds near the maritime continents and the Atlantic side of the American continent are in view (see Jiang et al. 2018). The reflected MESSENGER fluxes reported by García Muñoz (2015) show the same dependence of $F$ on $\lambda$, but a less obvious stronger peakto-peak variability with increasing wavelength. This might be explained by the smaller wavelength range that is covered by the MESSENGER data, as only 480, 560, and $630 \mathrm{~nm}$ are shown by García Muñoz (2015).

\subsection{Color changes}

In Fig. 5 we show the colors of our cloudy model planet (with an average cloud fraction of about 66\%) in $F, Q$, and $P$ as functions of the geographic longitude in the middle of the longitude range on the illuminated and visible part of the planetary disk, and phase angle $\alpha$ (these are functions of time themselves, i.e., with increasing longitude, $\alpha$ also slightly increases), computed using the curves at 443, 550, and $670 \mathrm{~nm}$ in Fig. 4. With increasing $\alpha$, the effect of the local patterns on the planet starts to appear, as the illuminated and visible part of the planetary disk decreases.

Figure 5 shows how the planet changes color in $F$ from blue through white to red with increasing $\alpha$. The planet appears white when the phase curves of $F$ at $\lambda=443,550$ and $670 \mathrm{~nm}$ intersect (cf. Fig. 4), which is near $\alpha=120^{\circ}$, in Fig. 5. The faint white vertical stripe near $\alpha=40^{\circ}$ is due to the primary rainbow. This rainbow feature is much stronger in $Q$ and $P$. Flux $F$ shows a few whitish, horizontal streaks that are related to the presence of continents around those longitudes, in particular, Africa (around $30^{\circ}$ ) and the Americas (around $300^{\circ}$ ). At smaller phase angles, the brown and green color of the arid and vegetated regions comprising the largest regions of the continents (see Fig. 2) compensate for the blueness of the atmosphere, while at the larger phase angles, the continents limit the reflection by the ocean that tends to color a planet red at these phase angles (Trees \& Stam 2019).

The color changes in polarized flux $Q$ and degree of polarization $P$ appear to be related to the visibility of an ocean surface depending on which part of the planet is visible to the observer. Trees \& Stam (2019) have derived that for ocean planets, that is, planets without continents, the color change phase angle for $F$ decreases with increasing cloud coverage fraction $f_{\mathrm{c}}$, while it increases with increasing $f_{\mathrm{c}}$ for $Q$ and $P$. In Fig. 5, $F$ changes color in a $5-10^{\circ}$ wide phase-angle range around $120^{\circ}$, which on an ocean planet would be indicative for $f_{\mathrm{c}}$ ranging from 0.25 to 


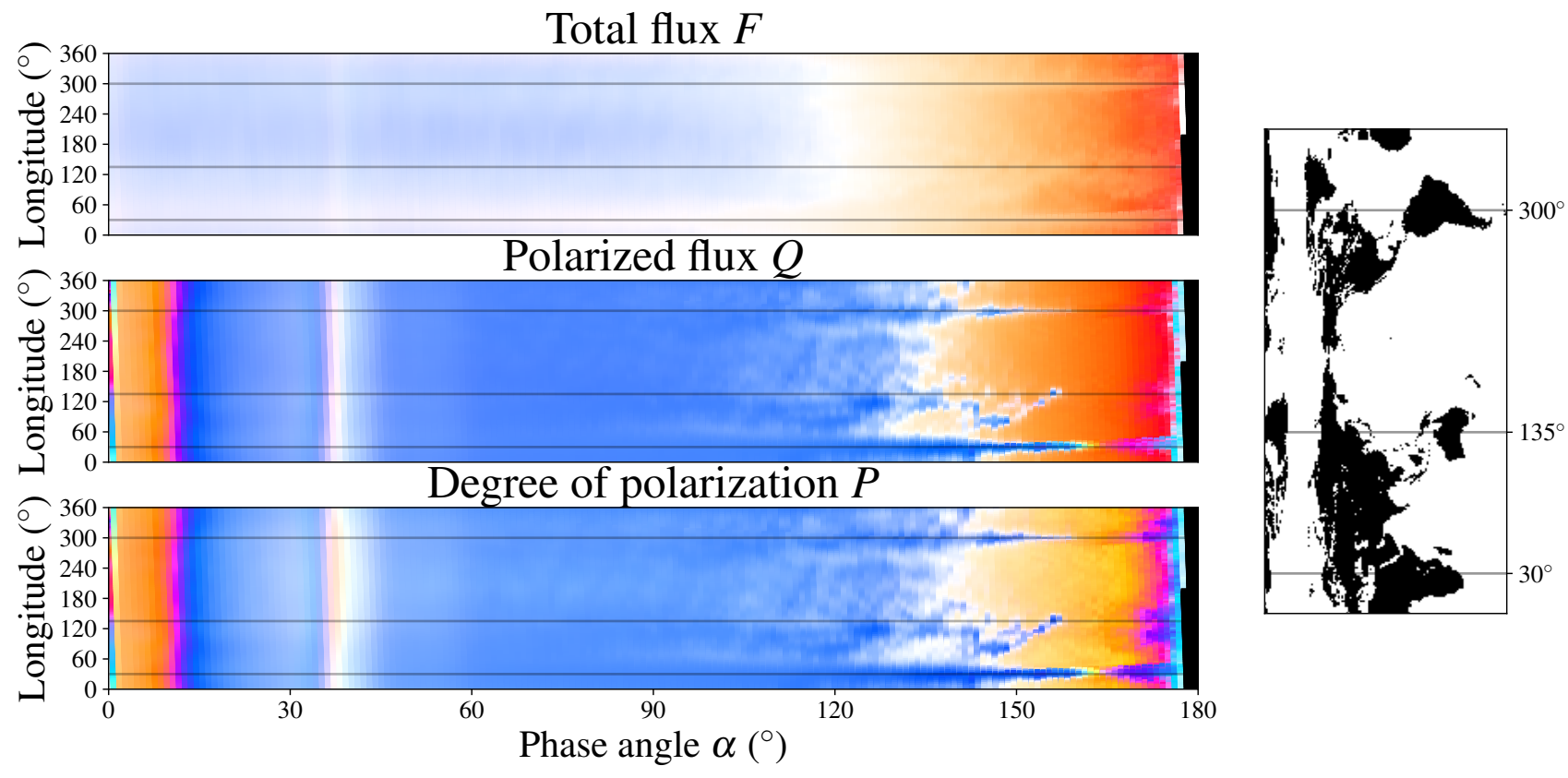

Fig. 5. RGB colors of $F$ (top), $Q$ (middle), and $P$ (bottom) of the cloudy model planet as functions of phase angle $\alpha$ and the longitude in the middle of the longitude range that is visible to the observer $\left(0^{\circ}\right.$ longitude corresponds with the Greenwich meridian). These color plots were constructed from the $F, Q$, and $P$ phase curves at 443, 550 and $670 \mathrm{~nm}$ as presented in Fig. 4. The horizontal black lines indicate the respective longitudes on the geographical map on the right.

0.75 (Trees \& Stam 2019). However, on these model planets, the continental surface coverage and the surface albedo of the continents also affects the color of $F$. According to Trees \& Stam (2019), for $Q$ and $P$ of ocean planets, the phase angle where the color changes increases with increasing $f_{\mathrm{c}}$, and Fig. 5 shows that adding continents (partly) below the clouds appears to have a similar effect: for longitudes with more continental coverage, the color-changing phase angle is larger than for longitudes with less continental coverage (assuming that the clouds are more or less evenly distributed across the planet).

A tentative color crossing in polarized Earth-shine observations has recently been presented by Sterzik et al. (2019). These measurements were obtained with ESO's Very Large Telescope (VLT). Given the telescope location in Chile, either the Pacific Ocean or South America and the Atlantic Ocean would have been oriented toward the Moon, depending on the time of the observations. The Pacific Ocean observations cover the widest phase-angle range (from about $50^{\circ}$ to $135^{\circ}$ ) and show a tentative color crossing near $135^{\circ}$. This phase angle is similar to what is shown in Fig. 5. The Atlantic Ocean observations cover phase angles from about $65^{\circ}$ to $116^{\circ}$, which is not far enough to actually show a color-crossing phase angle, but extrapolation by eye suggests a crossing phase-angle between $125^{\circ}$ and $130^{\circ}$, which would be smaller than shown in Fig. 5. This might be due to a different orientation of the Earth than used in our simulations or to different cloud properties. The precise relation between the color-changing phase angle, the surface coverage, and the (variable) cloud coverage and its applicability in characterizing the distribution of continents and ocean on exoplanets will be subject of a later study.

\section{Summary and discussion}

We have presented numerically computed total and polarized fluxes of starlight that is reflected by an Earth-like model planet as functions of time, the planetary phase angle, and for five different wavelengths in the continuum: $350,443,550,670$, and $865 \mathrm{~nm}$. These fluxes and the derived degree of polarization can play a crucial role as test data to help the design and development of astronomical instrumentation and observational strategies for the direct detection of small Earth-like exoplanets, especially because such data (across time, phase angles, and wavelength) are unavailable from Earth-observation missions.

Estimates show that with a dedicated technology maturation path, strategic exo-Earth characterization missions might be possible around 2030-2035 (Crill \& Siegler 2017). This estimate is based on an extensive study of current technology areas that must be advanced to image exo-Earths, albeit including only brightness (total flux) measurement techniques. Similarly, Feng et al. (2018) estimated the science return of space observatories such as HabEx and LUVOIR for different instrument designs with an inverse-modeling framework. They also considered only total fluxes.

With our results, the advantages of including spectropolarimetry can be studied on the instrument and mission level. In particular, our results can be used to investigate the effect of the polarimetric accuracy that can be achieved by different polarimetric techniques in an instrument and by different telescope designs, especially the effects of various advanced adaptive optics techniques, and different types of coronagraphs to reduce the background stellar flux and to optimize the inner working angle. By integrating our results over time, effects of observational integration times can be investigated.

Specific phase-angle-dependent features that would be worth detecting appear to be the primary rainbow as a tool for detecting liquid water clouds (as earlier identified, e.g., by Karalidi et al. 2012b; Bailey 2007), which is a stronger signal in $Q$ and $P$ than in $F$, the color-crossing phase angle in $Q$ or $P$ as a tool for detecting liquid surfaces (for details, see Trees \& Stam 2019), and the regular variations due to the daily rotation of the planet and the variability on those variations due to the changing cloud cover. When the orientation of the planetary light equator with 
respect to the instrumental optical plane is not known, because the orientation of the planetary orbit is not known, the distribution of linearly polarized flux over $Q$ and $U$ is generally different than in our results. The very low values of $|U|$ as compared to $|Q|$ when the instrumental reference plane is parallel to the light equator, even for a horizontally inhomogeneous planet like the Earth, could be used to determine the orientation of the light equator, and with this, of the orbit.

Because the diurnal variability of the signals appears to be little dependent on the wavelength, except for $|Q|$ at short wavelengths, broadband observations would help increase the signal-to-noise ratio (S/N) of observations (see Bailey 2007, for a similar discussion on the observability of the rainbow feature). At short wavelengths $(\lambda=350 \mathrm{~nm}$ in our results $)$, the variability of $|Q|$ strongly depends on the cloud properties and very little on the surface properties. Measuring in a short and a long wavelength band would therefore allow for uncoupling cloud and surface reflection. The color-crossing $\alpha$ for liquid surface detection is at a relatively large phase angle, that is, near $145^{\circ}$ for an Earth-like cloud coverage fraction (the larger the coverage fraction, the larger the color-crossing phase angle, see Trees \& Stam 2019), and $F,|Q|$ and $P$ are relatively small at these phase angles. In addition, the color of the planet should be determined, and observations through more than one spectral band should therefore be performed. However, the color-crossing can be detected across a range of phase angles of $10^{\circ}-20^{\circ}$ wide, which would allow for long integration times, see Fig. 5.

Our polarized flux results are valuable test data not only for spectropolarimeters, instruments designed to measure polarization, but also for spectrometers, instruments that might not have been designed to measure polarization, but that are often polarization sensitive due to the use of optical elements such as mirrors and gratings. Without a proper validation of the design of a spectrometer for incident light that is polarized, such as starlight that is reflected by an exoplanet, measurement errors arise that are hard to account for. When test data are used in combination with instrument simulators, the errors for a given design can be estimated and the design can be adapted to minimize them.

Because the physical parameters of our model Earth are known, our computed fluxes and the degree of polarization across time, phase angle, and wavelength can also be used to develop and/or test data analysis algorithms to be used on (future) measurements of light reflected by exoplanets, such as autocorrelation methods of the Fourier analysis to derive the planetary rotational period and/or the presence of continents (see Visser \& van de Bult 2015; Oakley \& Cash 2009; Pallé et al. 2008). To simulate actual measurements, our total and polarized fluxes would have to be scaled to those of the target exoplanet and its star, according to Eq. (7), noise sources (photon noise and background stellar light) and instrumental effects (e.g. derived from a preliminary design) could be added, by scaling the computed fluxes and/or integrating across instrument response functions, effects of different integration times and/or spectral resolutions can be studied. Our computed total and polarized fluxes could also be combined with total and polarized fluxes of light of the parent star that is scattered by exo-zodiacal dust (see, e.g., Renard et al. 1995) to investigate the effect of such local background sources on the detectability of the planetary signals.

For our model planet, we used Earth-observation data covering the period from January 1, 2011, to December 31, 2011, to describe the surface coverage and local albedos, the cloud coverage fraction, optical thickness, top pressure, and the effective radius of the cloud particles. The surface types that we took into account are oceans (with waves), vegetation (deciduous forest, grass, and steppe), arid regions (desert and shrublands), and ice or snow. The reflection by the ocean and arid regions fully includes linear (and circular) polarization, while that by the vegetation only includes linear polarization. Ice and snow are described by a Lambertian, that is, isotropic and unpolarized.

For the numerical results presented here, we did not include scattering and absorption by aerosol particles (small, suspended particles in the atmosphere): although numerically they are straightforward to include, their variations in space and time would require the addition of many atmosphere-surface models and hence severely increase the computing times. Zugger et al. (2011, 2010) investigated the effects of spherical maritime (sea-salt) aerosol particles (which are likely nonspherical, see Mishchenko et al. 2000) on the polarized reflection of ocean planets and concluded that depending on the visibility, these aerosols could significantly depolarize the reflected fluxes, although a comparison between the Earth-shine observations and model computations without aerosol presented by Sterzik et al. (2019) strongly suggests that aerosols in general have a minor effect on the polarized appearance of Earth, especially when compared to the effects of clouds (which Zugger et al. 2011, 2010 did not combine with the maritime aerosol). Our model atmospheres do not include (nonspherical) ice cloud particles either; all cloud particles are liquid water droplets. Scattering by nonspherical ice cloud particles was included by Karalidi et al. (2012b), who used both liquid water clouds and ice clouds in their model atmospheres. The ice- and liquidcloud optical thicknesses were derived from MODIS data. They adopted imperfectly shaped hexagonal ice-cloud particles (Hess 1998), and concluded among others, that the presence of ice clouds does not limit the visibility of the rainbow (which is due to spherical particles alone) in the polarization. Emde et al. (2017) used both types of cloud particles and relatively high ice-cloud optical thicknesses in their analysis of the polarized Earth-shine data published by Sterzik et al. (2012).

Our numerical computations do not include the absorption of light by atmospheric gases either, such as water and oxygen. This absorption can be included and will lead to features with high spectral resolution polarization across the absorption bands (see, e.g., Fauchez et al. 2017; Stam 2008), so that our fluxes should be considered to be representative of the continuum. Absorption by ozone is also neglected. This would be important to be included when wavelengths below $350 \mathrm{~nm}$ are of interest. The wavelength of $550 \mathrm{~nm}$ falls within a broad diffuse ozone absorption band (the Chappuis band) that would lower the reflected total and polarized fluxes, especially in the presence of clouds, but it would hardly change the degree of polarization (see, e.g., Stam 2008). We also ignored rotational Raman scattering, which is an inelastic scattering process by gaseous molecules, which would leave imprints of the stellar Fraunhofer lines in the total and polarized fluxes and in the degree of polarization as functions of the wavelength. In Earth observations, this phenomenon is usually referred to as the Ring effect after one of the discoverers (Grainger \& Ring 1962). Numerical simulations of rotational Raman scattering in atmospheres of gas giants (in total fluxes only) has been presented by Oklopčić et al. (2016). Rotational Raman scattering could be added to our numerical algorithm, but would require significant amounts of computing time.

Some improvements of our descriptions of the surface reflection are the reflection by the surface below shallow waters, altitude variations on the surface, such as mountains, regions of the ocean covered by sea ice, and seasonal variations in the 
surface reflection. Regarding the latter, in particular, seasonal albedo changes due to the migration of sea ice over the ocean, for instance, changes in the colors of vegetation, in the bloom of algae, etc. could be implemented. Polarization measurements of locally scattered and/or reflected light, for example, by instruments on board satellites in low Earth orbits, would be very useful for the extension and/or improvement of the currently missing or insufficient numerical descriptions of scattered or reflected light, as described above.

While validation of computed disk-integrated total and/or polarized fluxes reflected by a model Earth can be made somewhat using (polarized) Earth-shine measurements (see Sterzik et al. 2012), this method has several limitations, in particular, the unknown influence of the reflection of Earth-light by the lunar surface. While we can in principle measure how the surface (at different locations on the Moon) reflects incident (unpolarized) sunlight for various local solar zenith angles and various local viewing angles, we do not know how the surface reflects polarized light, especially not as a function of wavelength and covering a range of illumination and viewing geometries. Sterzik et al. (2019) presented a method to correct their polarized Earthshine measurements for the lunar depolarization effect, but the uncertainty in this method appears to be too large to validate parameter choices for the model planets. The ideal validation data would be spectropolarimetric measurements of the Earth taken from a distance. A compact instrument that would provide such data is the LOUPE (Hoeijmakers et al. 2016; Karalidi et al. 2012a), which aims to measure the total flux and the degree and direction of linear polarization from about 400 to $800 \mathrm{~nm}$ as the payload of a lunar lander or a geostationary satellite, for example (the latter would, however, not allow monitoring signal variations because the planet rotates daily). The spectropolarimeter LSDpol also aims to measure circular polarization across a similar spectral range 350-900 $\mathrm{nm}$ (Snik et al. 2019).

The computed total and polarized fluxes are available through the database researchdata.4tu.nl. They are archived under "Colors of an Earth-like exoplanet" with DOI 10.4121/ uuid:caa03e1a-0f6a-43e5-b67a-fa6f21829b8a

Acknowledgements. The MODIS Land Cover Type Data set (2011) is main tained by the NASA EOSDIS Land Processes Distributed Active Archive Center (LP DAAC) at the USGS/Earth Resources Observation and Science (EROS) Center. The Aqua/MODIS and Terra/MODIS Cloud Top Pressure, Cloud Particle Effective Radius, Cloud Optical Thickness and Cloud Fraction Daily L3 Global 1 Deg. CMG datasets were acquired from the Level-1 and Atmosphere Archive \& Distribution System (LAADS) Distributed Active Archive Center (DAAC), from the Goddard Space Flight Center in Greenbelt, Maryland, USA (https://1adsweb.nascom.nasa.gov/). The CCMP Version-2.0 vector wind analyses are produced by Remote Sensing Systems, with data available at www . remss . com. We acknowledge the use of the SCIAMACHY surface LER database provided by the Royal Netherlands Meteorological Institute (KNMI) L.R. acknowledges funding through the Planetary and Exoplanetary Science (PEPSci) Programme of the Netherlands Organisation for Scientific Research (NWO).

\section{References}

Aizawa, M., Kawahara, H., \& Fan, S. 2020, ApJ, 896, 22

ARM-facility 1998, Surface Spectral Albedo (SURFSPECALB1MLAWER). 2011/06/20 to 2011/06/20, Southern Great Plains (SGP) Central Facility, Lamont, OK (C1). Compiled by K. Gaustad and L. Riihimaki. Atmospheric Radiation Measurement (ARM) Data Center, http://dx.doi.org/10. $5439 / 1095394$

Atlas, R., Hoffman, R. N., Ardizzone, J., et al. 2011, Bull. Am. Meteorol. Soc., 92, 157

Bailey, J. 2007, Astrobiology, 7, 320

Baldridge, A. M., Hook, S. J., Grove, C. I., \& Rivera, G. 2009, Remote Sens. Environ., 113, 711
Bazzon, A., Schmid, H. M., \& Gisler, D. 2013, A\&A, 556, A117

Bean, J. L., Miller-Ricci Kempton, E., \& Homeier, D. 2010, Nature, 468, 669 Berdyugina, S. V., \& Kuhn, J. R. 2019, AJ, 158, 246

Berdyugina, S. V., Berdyugin, A. V., Fluri, D. M., \& Piirola, V. 2011, ApJ, 728, L6

Bétrémieux, Y., \& Kaltenegger, L. 2014, ApJ, 791, 7

Bouret, J.-C., Neiner, C., Gómez de Castro, A. I., et al. 2018, SPIE Conf. Ser., 10699, 106993B

Bryant, H. C., \& Jarmie, N. 1974, Sci. Am., 231, 60

Chou, F., Johnson, M., \& Landau, E. 2017, NASA Releases Kepler Survey Catalog with Hundreds of New Planet Candidates, https://www.nasa. gov/

Chowdhary, J., Cairns, B., \& Travis, L. D. 2006, Appl. Opt., 45, 5542

Ciddor, P. E. 1996, Appl. Opt., 35, 1566

Coakley, J. 2003, Reflectance and Albedo Surface (New York: Academic Press), 1914

Cotton, D. V., Marshall, J. P., Bailey, J., et al. 2017, MNRAS, 467, 873

Cox, C., \& Munk, W. 1954, J. Opt. Soc. Am. 1917-1983, 44, 838

Crill, B. P., \& Siegler, N. 2017, SPIE Conf. Ser., 10398, 103980H

de Haan, J. F., Bosma, P. B., \& Hovenier, J. W. 1987, A\&A, 183, 371

De Rooij, W. A., \& van der Stap, C. C. A. H. 1984, A\&A, 131, 237

Deschamps, P. Y., Breon, F. M., Leroy, M., et al. 1994, IEEE Trans. Geosci. Remote Sens., 32, 598

Dubovik, O., Li, Z., Mishchenko, M. I., et al. 2019, J. Quant. Spectr. Rad. Transf., 224, 474

Emde, C., Buras-Schnell, R., Sterzik, M., \& Bagnulo, S. 2017, A\&A, 605, A2

Fan, S., Li, C., Li, J.-Z., et al. 2019, ApJ, 882, L1

Fauchez, T., Rossi, L., \& Stam, D. M. 2017, ApJ, 842, 41

Feng, Y. K., Robinson, T. D., Fortney, J. J., et al. 2018, AJ, 155, 200

Ford, E. B., Seager, S., \& Turner, E. L. 2001, Nature, 412, 885

Fougnie, B., Bracco, G., Lafrance, B., et al. 2007, Appl. Opt., 46, 5435

Friedl, M., \& Sulla-Menashe, D. 2019, MCD12Q1 MODIS/Terra+Aqua Land Cover Type Yearly L3 Global 500m GEO Grid V006. NASA EOSDIS Land Processes DAAC, https://doi .org/10.5067/MODIS/MCD12C1.006

Fujii, Y., Kawahara, H., Suto, Y., et al. 2011, ApJ, 738, 184

García Muñoz, A. 2015, Int. J. Astrobiol., 14, 379

García Muñoz, A., Zapatero Osorio, M. R., Barrena, R., et al. 2012, ApJ, 755, 103

Gilbert, E. A., Barclay, T., Schlieder, J. E., et al. 2020, AJ, submitted, [arXiv:2001.00952]

Ginoux, P., Chin, M., Tegen, I., et al. 2001, J. Geophys. Res., 106, 255

Ginski, C., Benisty, M., van Holstein, R. G., et al. 2018, A\&A, 616, A79

Grainger, J. F., \& Ring, J. 1962, Nature, 193, 762

Hale, G. M., \& Querry, M. R. 1973, Appl. Opt., 12, 555

Hall, D. K., \& Riggs, G. A. 2016, MODIS/Aqua Snow Cover Daily L3 Global $500 \mathrm{~m}$ SIN Grid, Version 6. MYD10C1. Boulder, Colorado USA. NASA National Snow and Ice Data Center Distributed Active Archive Center, https://doi.org/10.5067/MODIS/MOD10A1.006

Han, Q., Rossow, W. B., \& Lacis, A. A. 1994, J. Clim., 7, 465

Hansen, J. E., \& Travis, L. D. 1974, Space Sci. Rev., 16, 527

Hess, M. 1998, J. Quant. Spectr. Rad. Transf., 60, 301

Hoeijmakers, H. J., Arts, M. L. J., Snik, F., et al. 2016, Opt. Express, 24, 21435

Hovenier, J. W., \& van der Mee, C. V. M. 1983, A\&A, 128, 1

Hovenier, J. W., Van Der Mee, C., \& Domke, H. 2004, Astrophys. Space Sci. Lib., 318

Hubanks, P., Platnick, S., King, M., \& Ridgway, B. 2015, ATBD reference number ATBD-MOD-30, NASA, 125

Jiang, J. H., Zhai, A. J., Herman, J., et al. 2018, AJ, 156, 26

Kaltenegger, L., \& Traub, W. A. 2009, ApJ, 698, 519

Karalidi, T., Stam, D. M., \& Hovenier, J. W. 2011, A\&A, 530, A69

Karalidi, T., Stam, D. M., Snik, F., et al. 2012a, Planet. Space Sci., 74, 202

Karalidi, T., Stam, D. M., \& Hovenier, J. W. 2012b, A\&A, 548, A90

Kasper, M., Beuzit, J.-L., Verinaud, C., et al. 2010, SPIE Conf. Ser., 7735, $77352 \mathrm{E}$

Kawahara, H., \& Fujii, Y. 2011, ApJ, 739, L62

Kawata, Y. 1978, Icarus, 33, 217

Keller, C. U., Schmid, H. M., Venema, L. B., et al. 2010, SPIE Conf. Ser., 7735, $77356 \mathrm{G}$

Kemp, J. C., Henson, G. D., Steiner, C. T., Beardsley, I. S., \& Powell, E. R. 1987, Nature, 328, 92

Kiang, N. Y., Segura, A., Tinetti, G., et al. 2007a, Astrobiology, 7, 252

Kiang, N. Y., Siefert, J., Govindjee, \& Blankenship, R. E. 2007b, Astrobiology, 7, 222

King, M. D., Closs, J., Spangler, S., et al. 2004, EOS Data Products Handbook Volume 1, https://eospso.nasa.gov/sites/default/files/ publications/data_products_1.pdf

Koepke, P. 1984, Appl. Opt., 23, 1816

Kostogryz, N. M., Yakobchuk, T. M., \& Berdyugina, S. V. 2015, ApJ, 806, 97 
Maignan, F., Bréon, F.-M., Fédèle, E., \& Bouvier, M. 2009, Remote Sens Environ., 113, 2642

Mayor, M., \& Queloz, D. 1995, Nature, 378, 355

Meerdink, S. K., Hook, S. J., Roberts, D. A., \& Abbott, E. A. 2019, Remote Sens. Environ., 230, 111196

Miles-Páez, P. A., Pallé, E., \& Zapatero Osorio, M. R. 2014, A\&A, 562, L5

Millar-Blanchaer, M. A., Girard, J. H., Karalidi, T., et al. 2020, ApJ, 894, 42

Mishchenko, M. I., \& Travis, L. D. 1997, J. Geophys. Res., 102, 16989

Mishchenko, M. I., Hovenier, J. W., \& Travis, L. D. 2000, Light scattering by nonspherical particles : theory, measurements, and applications (San Diego: Academic Press)

Misra, A., Meadows, V., \& Crisp, D. 2014, ApJ, 792, 61

Montañés-Rodríguez, P., Pallé, E., Goode, P. R., \& Martín-Torres, F. J. 2006 ApJ, 651, 544

Morel, A. 1974, Opt. Aspects Oceanogr., 1, 1

Moreno, F., Vilaplana, R., Muñoz, O., Molina, A., \& Guirado, D. 2006, J. Quant. Spectr. Rad. Transf., 100, 277

Muñoz, O., Moreno, R., Guirado, D., et al. 2012, Digital Handbook. The 2012 European Aerosol Conference, 53

Nadal, F., \& Breon, F. M. 1999, IEEE Trans. Geosci. Remote Sens., 37, 1709

Nakajima, T. 1983, J. Quant. Spectr. Rad. Transf., 29, 521

Nakajima, T., \& King, M. D. 1990, J. Atm. Sci., 47, 1878

Oakley, P. H. H., \& Cash, W. 2009, ApJ, 700, 1428

Oklopčić, A., Hirata, C. M., \& Heng, K. 2016, ApJ, 832, 30

Oreopoulos, L., Cahalan, R. F., \& Platnick, S. 2007, J. Clim., 20, 5114

Pallé, E., Ford, E. B., Seager, S., Montañés-Rodríguez, P., \& Vazquez, M. 2008, ApJ, 676, 1319

Parkinson, C. L., \& Greenstone, R. 2000, EOS Data Products Handbook Volume 2, https://eospso.gsfc.nasa.gov/sites/default/files/ publications/data_products_vol2_0.pdf

Patty, C. H. L., ten Kate, I. L., Buma, W. J., et al. 2019, Astrobiology, 19, 1221

Perryman, M. 2018, The Exoplanet Handbook (Cambridge: Cambridge University Press)

Platnick, S., et al. 2015, MODIS Atmosphere L3 Daily Product. NASA MODIS Adaptive Processing System, Goddard Space Flight Center, USA, http: // dx.doi.org/10.5067/MODIS/MYD08_D3.006

Pope, R. M., \& Fry, E. S. 1997, Appl. Opt., 36, 8710

Renard, J. B., Levasseur-Regourd, A. C., \& Dumont, R. 1995, A\&A, 304, 602

Robinson, T. D., Meadows, V. S., Crisp, D., et al. 2011, Astrobiology, 11, 393

Rodriguez, J. E., Vanderburg, A., Zieba, S., et al. 2020, AJ, submitted [arXiv:2001.00954]

Rossi, L., \& Stam, D. M. 2018, A\&A, 616, A117

Rossi, L., Berzosa-Molina, J., \& Stam, D. M. 2018, A\&A, 616, A147
Roujean, J.-L., Leroy, M., \& Deschamps, P.-Y. 1992, J. Geophys. Res., 97, 20, 455

Schaepman-Strub, G., Schaepman, M. E., Painter, T. H., Dangel, S., \& Martonchik, J. V. 2006, Remote Sens. Environ., 103, 27

Schwieterman, E. W., Kiang, N. Y., Parenteau, M. N., et al. 2018, Astrobiology, 18,663

Seager, S., Whitney, B. A., \& Sasselov, D. D. 2000, ApJ, 540, 504

Seager, S., Turner, E. L., Schafer, J., \& Ford, E. B. 2005, Astrobiology, 5, 372

Smith, R. C. \& Baker, K. S. 1981, Appl. Opt., 20, 177

Snik, F., Keller, C. U., Doelman, D. S., et al. 2019, SPIE Conf. Ser., 11132, $111320 \mathrm{~A}$

Sogandares, F. M., \& Fry, E. S. 1997, Appl. Opt., 36, 8699

Sparks, W. B., Hough, J. H., Kolokolova, L., et al. 2009, J. Quant. Spectr. Rad. Transf., 110, 1771

Stam, D. M. 2008, A\&A, 482, 989

Sterzik, M. F., Bagnulo, S., \& Palle, E. 2012, Nature, 483, 64

Sterzik, M. F., Bagnulo, S., Stam, D. M., Emde, C., \& Manev, M. 2019, A\&A, 622, A41

Swain, M. R., Vasisht, G., \& Tinetti, G. 2008, Nature, 452, 329

Swain, M. R., Tinetti, G., Vasisht, G., et al. 2009, ApJ, 704, 1616

Tilstra, L. G., Tuinder, O. N. E., Wang, P., \& Stammes, P. 2017, J. Geophys. Res. Atmos., 122, 4084

Tinetti, G., Vidal-Madjar, A., Liang, M.-C., et al. 2007, Nature, 448, 169

Trees, V. J. H., \& Stam, D. M. 2019, A\&A, 626, A129

Tsang, L., Kong, J. A., \& Shin, R. T. 1985, Theory of Microwave Remote Sensing (New York: Wiley Interscience)

Turnbull, M. C., Traub, W. A., Jucks, K. W., et al. 2006, ApJ, 644, 551

Visser, P. M., \& van de Bult, F. J. 2015, A\&A, 579, A21

Wang, S., Qu, Z.-Q., \& Li, H. 2019, Res. A\&A, 19, 117

Wentz, F. J., Scott, R., Hoffman, R., et al. 2015, Remote Sensing Systems Cross-Calibrated Multi-Platform (CCMP) 6-hourly ocean vector wind analysis product on $0.25 \mathrm{deg}$ grid, Version 2. 0. Remote Sensing Systems, Santa Rosa, CA., http: //www . remss . com/measurements/ccmp

Wiktorowicz, S. J., \& Stam, D. M. 2015, Exoplanets (Cambridge: Cambridge University Press), 439

Williams, D. M., \& Gaidos, E. 2008, Icarus, 195, 927

Woolf, N. J., Smith, P. S., Traub, W. A., \& Jucks, K. W. 2002, ApJ, 574 430

Zhai, P.-W., Hu, Y., Chowdhary, J., et al. 2010, J. Quant. Spectr. Rad. Transf., 111,1025

Zugger, M. E., Kasting, J. F., Williams, D. M., Kane, T. J., \& Philbrick, C. R. 2010, ApJ, 723, 1168

Zugger, M. E., Kasting, J. F., Williams, D. M., Kane, T. J., \& Philbrick, C. R. 2011, ApJ, 739, 55 